\title{
Light Threshold Effects in Supersymmetric Grand Unified Theories.
}

\author{
Alon E. Faraggi ${ }^{\ddagger}$ \\ Department of Physics, Weizmann Institute of Science \\ Rehovot 76100, Israel \\ and \\ Benjamín Grinstein ${ }^{\dagger}$ \\ Superconducting Super Collider Laboratory \\ 2550 Beckleymeade Ave, Dallas, Texas 75237, USA
}

Supersymmetric Grand Unified Theories have a rich spectrum of particles barely heavier than the intermediate vector bosons. As their non-supersymmetric counterparts, they lead to many relations among low energy observables. But the precise form of the predictions is modified by the extended spectrum. If the masses of these new particles are comparable to $M_{Z}$, the standard computation of their effect becomes inaccurate. We present a detail discussion of the correct procedure, and carry out the relevant computations to one loop order. Attention is paid to the special treatment that the top and Higgs particles must receive. The size of the effect is explored for a range of parameters in the minimal supersymmetric $S U(5)$ grand-unified theory with radiative breaking. It is found that the naive (leading-log) computation can be fairly inaccurate.

August 1993

¥ fhalon@weizmann. Address after September 1 1993, School of Natural Sciences, Institute for Advanced Study, Olden Lane, Princeton NJ, 08540.

$\dagger$ grinstein@sscvx1.bitnet, @sscvx1.ssc.gov 


\section{DISCLAIMER}

This report was prepared as an account of work sponsored by an agency of the United States Government. Neither the United States Government nor any agency thereof, nor any of their employees, makes any warranty, express or implied, or assumes any legal liability or responsibility for the accuracy, completeness, or usefulness of any information, apparatus, product, or process disclosed, or represents that its use would not infringe privately owned rights. Reference herein to any specific commercial product, process, or service by trade name, trademark, manufacturer, or otherwise does not necessarily constitute or imply its endorsement, recommendation, or favoring by the United States Government or any agency thereof. The views and opinions of authors expressed herein do not necessarily state or reflect those of the United States Government or any agency thereof. 


\section{DISCLAIMER}

Portions of this document may be illegible in electronic image products. Images are produced from the best available original document. 


\section{Introduction}

Grand Unified Theories possess many attractive features. They simplify enormously the description of matter content of the standard model by reducing the number of irreducible representations of the gauge group needed to account for all observed particles. They explain electric charge quantization. They bring all non-gravitational interactions under a common umbrella treatment. Yet, the minimal standard model extension to an SU(5) GUT is all but ruled out: not only does proton not decay at the predicted rate, precise measurements[1] of the gauge couplings of strong and electroweak interactions have led to the observation that at no scale do they unify.

This rather grim observation is, nevertheless, not generic of GUT theories, and it seems premature to dismiss the class of theories on the basis of the failure of the minimal version. Remarkably, the minimal supersymmetric extension of the minimal SU(5) GUT is free of the aforementioned problems. That the three gauge couplings unify[2] in that case is not a surprise: an additional parameter, $M_{\mathrm{SUSY}}$, is introduced which can be chosen so that couplings do unify. What is remarkable is that unification occurs at a scale $M_{\mathrm{GUT}}$ high enough that proton stability is not in conflict with observation, yet bellow the Planck scale, making it plausible that calculations with presently understood techniques are sensible. Moreover, the additional parameter, $M_{\mathrm{SUSY}}$, which describes the scale at which the running of the gauge couplings changes from what is dictated by the standard model to what is dictated by its supersymmetric extension, comes out rather small, just about equal to the scale of electroweak symmetry breaking. If one interprets $M_{\text {SUSY }}$ as some average mass of the supersymmetric partners of standard particles, the inescapable conclusion is that a rich spectrum of new elementary particles is awaiting discovery in the few hundred $\mathrm{GeV}$ range (well accessible to planned next generation hadron colliders).

One ought not to rush into conclusions, though. Barbieri and Hall[3] have pointed out that the standard analysis of running of gauge couplings assumes all the superheavy particles (those with masses naturally of order of $M_{\mathrm{GUT}}$ ) are degenerate. The value of $M_{\text {SUSY }}$, they argue, can be shifted if one drops this assumption and includes the effects of the non-degenerate superheavy thresholds. On closer examination, it has been noticed[4] that in the minimal SUSY SU(5) model the dependence of $M_{\text {SUSY }}$ on superheavy thresholds is rather weak. 
For example, if $\alpha_{s}\left(M_{Z}\right)=0.125$ with small errors ${ }^{1}$, the value of $M_{\mathrm{SUSY}}$ exclusive of superheavy threshold effects comes out to be well below the electroweak scale. Superheavy thresholds can push $M_{\text {SUSY }}$ back up. But to obtain $M_{\text {SUSY }} \geq M_{Z}$ one must have that the superheavy supermultiplets that transform as $(8,1)_{0}+(1,3)_{0}$ under the gauge group $S U(3) \times S U(2) \times U(1)$ acquire masses some eight orders of magnitude below $M_{\mathrm{GUT}}$. Worse yet, to keep $M_{\mathrm{GUT}}$ well below the Planck scale one must further complicate the spectrum of superheavies, splitting the masses of these two multiplets by two orders of magnitude. The simple compelling picture of a dessert between the electroweak and GUT scales is lost. Instead one must populate the dessert with particles at two new intermediate scales. For the remaining of this work we neglect these heavy threshold effects.

In addition to superheavy threshold effects, one must consider the effects of light thresholds (non-degeneracy of the SUSY particles with masses naturally of order the electroweak scale). Ross and Roberts[5] argue that because in most models the average mass of the colored superpartners is larger than that of the uncolored ones, a simple parameterization in terms of a single threshold at $M_{\text {SUSY }}$ may be misleading. Langacker and Polonski[6] point out that one need not interpret $M_{\text {SUSY }}$ as some sort of average mass for the spectrum of SUSY particles. The spectrum of the model is not expected to be simple. One can imagine accounting for the complicated spectrum in some accurate fashion, and then finding a scale $M_{\text {SUSY }}$ which would mimic the effects of a complicated spectrum. Only this scale does not have a clear physical meaning. In fact, they find it possible to construct examples of rather heavy SUSY spectra (compared with $M_{Z}$ ), yet having $M_{\text {SUSY }} \ll M_{Z}$.

The standard analyses of light threshold effects consists of modifying the evolution equations for the gauge couplings at each consecutive threshold[7]. More specifically, starting from unified gauge couplings at $M_{\mathrm{GUT}}, \alpha_{1}\left(M_{\mathrm{GUT}}\right)=\alpha_{2}\left(M_{\mathrm{GUT}}\right)=\alpha_{3}\left(M_{\mathrm{GUT}}\right)$, one uses the renormalization group equation (RGE) to evolve these couplings down to the first threshold. The evolution equations are modified by reducing the number of active degrees freedom that affect the running, and the couplings are run further down to the next threshold, and so on. In what follows we shall refer to this method as the 'run-and-match' or the 'naive' method. This method is justified, and expected to be a good approximation, provided all of the SUSY particles are much heavier than the $Z$-boson. Also, this kind of analyses by necessity assumes unbroken electroweak symmetry, which again is reasonable at mass scáles well above $M_{Z}$.

1 A literature search shows that its value, as reported by LEP experiments over the last two years, has wandered over few times the quoted error bars. 
In this paper we address the issue of how to compute these light threshold effects when the SUSY particles are not necessarily much heavier than the $Z$-boson. The motivation for this comes not just from the discussion just given, but from the recent observation that phenomenologically viable models that incorporate SUSY SU(5) and radiative electroweak breaking invariably contain many particles with masses barely exceeding $M_{Z}$.

We will first formulate carefully the question we want to address. This is done in section 2 where we give the fundamental equations that allow us to relate the measurements of gauge couplings as reported by, say, LEP/SLC experiments to the gauge couplings of the SUSY model. In section 3 we develop general formulae for threshold effects modifications to relations among parameters dictated by grand unified theories. In section 4 we exhibit the result of one loop computations of the quantities that go into the general formulae of the preceding section. Special care must be taken in dealing with the Higgs sector and the top quark, and this is addressed in section 5. In preparation for the discussion and presentation of our numerical analysis of section 7 , we review the minimal supergravity $S U(5)$ model in section 6 . Our conclusions can be found in section 8 .

There is a long history of calculations of radiative corrections in and beyond the electroweak theory[8]. Calculations in supersymmetric theories date back to refs. [9]. No doubt the trivial calculations of one loop diagrams in this paper can be found elsewhere. The emphasis in this paper is on the treatment of light threshold effects in grand-unified theories, and in particular in SUSY-GUTs. It may not be obvious to the reader but, as we will see (sect. 2), the computations involved coincide with those of radiative corrections to electroweak parameters. Thus, for example, while ref. [10] presents a detailed discussion of radiative corrections to electroweak parameters in SUSY extensions of the standard model (see table 1 and figs. 39-45 of that work), the same work treats the case of grand-unified SUSY theories as containing a single common mass threshold at a scale ' $\mu$ ' (see figs. 47-49 there). We believe ours is therefore the most complete treatment of the light thresholds in SUSY GUTs to date. Moreover, we have failed to find as detailed a presentation of the numerical size of the effects for the minimal SUSY SU(5) GUT theory with radiative breaking as we give here ( $c f$, sect. 7$)$.

\section{Generalities}

Gauge coupling constants are not physical observables. When an experiment reports on the value of a gauge coupling, there is an implicit translation of some observables into 
these theoretical constructs. This is not to say that the values of gauge couplings lack in importance. On the contrary, given a well specified definition of these couplings, they encode concisely the results of measurements.

When an experimental result is analyzed to extract the value of gauge coupling constants, the actual value obtained depends in detail on the theoretical assumptions. Given two different models with the same gauge group but different particle content, as is the case of the standard model and its supersymmetric extension, the extracted values of gauge couplings from the same set of observables is a priori different for the two models. In principle one could analyze the observables directly under the different set of assumptions, $i e$, different models, and thus extract the values of gauge coupling constants appropriate to those assumptions. In practice however, it is impossible to reanalyze the experiment for each new set of assumptions.

The results of experiments are therefore most often given in terms of an analysis based on the standard model. This makes sense. The standard model is the most concise model of elementary interactions consistent with all present observations. Clearly what we need is a means for translating experimentally determined values of gauge couplings in the standard model into gauge couplings in extensions of the standard model.

It is possible to have such a translation. An obvious case is one in which the model at hand is an extension of the standard model that has only very heavy particles with hard masses. By this we mean that their masses are much larger than the energies at which the experiments are carried out, and that the masses are symmetric under the electroweak $S U(2) \times U(1)$, up to small corrections. Then the decoupling theorem guarantees that the physics at experimental energies is described by an effective Lagrangian which corresponds precisely to the standard model. The effective coupling constants of this effective theory are numerically equal to the standard model's. The gauge couplings of the underlying theory are easily related to those of the effective theory, by matching conditions at the scale of the heavy particles. Non-supersymmetric GUTs furnish an example. The underlying coupling constant, $\alpha_{G U T}$, can be derived by matching at $M_{\mathrm{GUT}}$, so one must determine experimentally the electroweak and strong couplings at a low scale, and then run them up to $M_{\mathrm{GUT}}$ where the effective theory can be matched to the full underlying theory.

In fact, this method can also be used even when the heavy particles are only moderately heavier than the energy scale of experiments. This is because this method accounts for all effects that are logarithmic in the ratio of the experimental scale $E$ and the heavy mass $M, \ln (E / M)$; corrections are suppressed by powers of $E / M$, or even of $(E / M)^{2}$. 
Therefore it is quite sensible to use this method to analyze SUSY GUTs, especially if one is interested in a spectrum of heavy particles in the $\mathrm{TeV}$ range. In fact, this is precisely the way in which the low threshold effects have been accounted for by many in the past $[5--7,11]$. We refer to this method as the 'run-and-match' or 'naive' calculation.

But one may ask what the proper procedure is, and even whether one exists, for the case in which the heavy particles are only barely heavier than the experimental scale, ie, $E / M$ is only slightly smaller than unity. After all, many recent analysis of SUSY GUTS show that models typically contain several particles in the few hundred $\mathrm{GeV}$ range. And not only is $E / M$ only slightly smaller than 1 ; since the multiplicity is high, one expects to find large coefficients in front of the order $(E / M)$ correction.

It turns out it is easy to construct the proper procedure. We will assume that particles are heavy enough that they cannot be directly produced at present (else, the theory should be ruled out or confirmed experimentally in short order!). This is important because it implies immediately that the only observables available are those of the standard model. In other words, we can consider, say, cross sections or decay widths of standard model particles for comparison with experiment, and not consider any novel processes of the extended theory. Let $O_{i}, i=1,2,3, \ldots$ stand for a collection of observables. For example, this can be taken to be the forward-backward asymmetry at the peak of the $Z$-resonance, the differential cross section $d \sigma\left(e^{+} e^{-} \rightarrow \mu^{+} \mu^{-}\right) / d \cos \theta$ at a collection of specific center of mass energies and angles, and similar cross sections into other leptons or quarks. The standard model gives expressions $F_{i}$ for these observables in terms of its parameters $g_{k}$,

$$
O_{i}=F_{i}\left(g_{k}\right) \text {. }
$$

Now, any extension of the standard model will also give expressions $\tilde{F}_{i}$ for the same observables in term of its own parameters. Among these parameters are those which have a direct correspondence to those of the standard model, $\tilde{g}_{k}$, like gauge couplings and particle masses. In addition there are new parameters $e_{n}$ characterizing the new physics, like new particle masses. So one has,

$$
O_{i}=\tilde{F}_{i}\left(\tilde{g}_{k}, e_{n}\right)
$$

Although conceptually the same, one must differentiate between the couplings $g_{k}$ and $\tilde{g}_{k}$. In fact, it is this difference that we are trying to calculate for the case of gauge couplings! 
In other words, the problem at hand is to calculate $\tilde{g}_{k}$ from knowledge of $g_{k}$, given an assumption on the values of the parameters $e_{n}$.

Our task is to invert eqs (2.1)-(2.2) for $\tilde{g}_{k}$ in terms of $g_{k}$ (and, implicitly, $e_{n}$ ). Now, the additional degrees of freedom enter into the expressions for observables as heavy virtual particles. Therefore, their effects are small. We can write

$$
\tilde{g}_{k}=g_{k}+\delta g_{k}
$$

where $\delta g_{k}$ are small one loop order corrections which are expressed as functions of $g_{k}$ (and, implicitly, $e_{n}$ ). It turns out to be convenient to write the functions $\tilde{F}$ as a sum of two pieces, one identical to the standard model's, plus the rest:

$$
\tilde{F}_{i}\left(\tilde{g}_{k}, e_{n}\right)=F_{i}\left(\tilde{g}_{k}\right)+\Delta F_{i}\left(\tilde{g}_{k}, e_{n}\right)
$$

This is convenient because $\Delta F$ can be regarded as a small quantity, the same order as $\delta g$. This means also that we are assuming the same renormalization scheme and gauge choice in both models. Combining equations and keeping only leading order in small quantities, one obtains

$$
\sum_{l} \delta g_{l} \frac{\partial F_{i}}{\partial g_{l}}\left(g_{k}\right)+\Delta F_{i}\left(g_{k}, e_{n}\right)=0
$$

Equation (2.5) is a set of simultaneous equations for $\delta g_{k}$. In the first term, the derivative $\frac{\partial F_{i}}{\partial g_{l}}$ should be evaluated at tree level, because the coefficient $\delta g_{l}$ is already of one loop order. This is a welcome simplification: we only need to compute the one loop effects of the new particles, because the standard ones have already been included in the experimental determination of the couplings $g_{k}$.

It is important to note that this discussion depends crucially on approximate decoupling. Were the new particles not heavy enough, the dependence of observables on kinematic variables (like $s$ and $t$ in cross sections) would differ from one model to the other. To the extent that deviations from the standard model cannot be inferred from kinematic dependence of observables one can neglect these kinematic effects in $\Delta F$. Although this observation seems rather innocuous, it is of practical importance. We need it to ensure compatibility of expressions (2.1) and (2.2).

Now, it would seem the program of determining the threshold corrections $\delta g_{k}$ is complete: calculate the functions $F_{i}$ at tree level, and the virtual effects of the new particles at one loop $\Delta F_{i}$, and plug into eq. (2.5). Then solve for $\delta g_{k}$. Still, this program involves a 
choice of specific observables, labeled by $i$. Recall that we would like to be able to connect the $\tilde{g}_{k}$ to the $g_{k}$ without detailed knowledge of the experimental data.

The Feynman diagrams that contribute to the functions $\Delta F_{i}$ can be classed in two groups: corrections to standard particle propagators and vertex or box corrections. The former are process independent, $i e$, they enter in a universal form into the relation between $\tilde{g}$ and $g$. The latter depend on each process, but are often smaller than the former. We therefore drop them. In standard lingo, we retain only oblique corrections. In any case this kind of truncation is implicitly done in the run-and-match approach. Our analysis is intended as an improvement on that approach, but it is clear that it is incomplete in this regard. (It should also be clear, from the above discussion, that short of a complete reanalysis of experimental data, this is the best one can do).

\section{Derivation of Master Formulas.}

We are ready to proceed with explicit calculations. Although the expression for the threshold corrections $\delta g_{k}$ will be process independent, it is necessary to choose particular (gedanken) process to obtain it.

We first consider a particularly simple example, $\mu \bar{\nu}_{\mu} \rightarrow e \bar{\nu}_{e}$ scattering. We hope this will serve to illustrate the general approach with a minimum of unnecessary complications. We take the observable $O$ as the cross section (at some kinematic point), divided by the phase space, $i e$, the square-modulus of the invariant amplitude,

$$
O=F(g)=\sum_{\text {helicities }}|\mathcal{A}|^{2}
$$

At tree level this is given by the Feynman diagram in fig. 1

Now, we really only need to extract the dependence on couplings, without paying careful attention to the kinematic factors. Therefore we write

$$
\sum_{\text {helicities }}|\mathcal{A}|^{2}=\left(\frac{g_{2}^{2}}{s-g_{2}^{2} v^{2}}\right)^{2} \sum_{\text {helicities }}|\tilde{\mathcal{A}}|^{2}
$$

Here $s$ is the standard kinematic variable, $g_{2}$ is the $S U(2)$ coupling constant and $v$ is the electroweak breaking vacuum expectation value. Clearly $\tilde{\mathcal{A}}$ is just a product of spinors and gamma matrices.

The one loop virtual effects of new heavy particles includes vertex, box and propagator corrections; these are shown in fig. 2. As discussed previously, we will neglect the vertex 
corrections. The propagator corrections are conveniently expressed in terms of the selfenergy (defined as the 1PI amputated two point function for the charged vector boson):

$$
\Pi_{W \mu \nu}(q)=\left(q^{2} g_{\mu \nu}-q_{\mu} q_{\nu}\right) \Pi_{W}^{(T)}+q_{\mu} q_{\nu} \Pi_{W}^{(L)}
$$

In terms of these the $W$-boson propagator in Feynman-'tHooft gauge is

$$
-i \frac{g_{\mu \nu}-q_{\mu} q_{\nu} / q^{2}}{q^{2}-g_{2}^{2} v^{2}-q^{2} \Pi_{W}^{(T)}}-i \frac{q_{\mu} q_{\nu} / q^{2}}{q^{2}-g_{2}^{2} v^{2}-q^{2} \Pi_{W}^{(L)}}
$$

The function $\Delta F$ that we need to compute is defined by eq. (2.4), which tells us that we should take the difference between $|\mathcal{A}|^{2}$ in the extended model and in the standard model. The tree level amplitudes are equal in both models, so the difference comes from the cross product of the tree level amplitude and the one-loop amplitude:

$$
\Delta F=2 g_{2}^{4} \operatorname{Re} \frac{s \hat{\Pi}_{W}^{(T)}}{\left(s-g_{2}^{2} v^{2}\right)^{3}} \sum_{\text {helicities }}|\tilde{\mathcal{A}}|^{2}
$$

Here by $\hat{\Pi}_{W}^{(T)}$ we mean that part of the self-energy which is not already in the standard model calculation, $i e$, which comes from the new particles of the extended model running around in the loop. Using eqs (3.1)-(3.5) in eq. (2.5), one obtains

$$
\delta g_{2} \frac{\partial}{\partial g_{2}}\left(\frac{g_{2}^{2}}{s-g_{2}^{2} v^{2}}\right)^{2}+\frac{2 g_{2}^{4} s}{\left(s-g_{2}^{2} v^{2}\right)^{3}} \operatorname{Re} \hat{\Pi}_{W}^{(T)}=0 .
$$

The solution is immediate:

$$
\delta g_{2}^{2}=-g_{2}^{2} \operatorname{Re} \hat{\Pi}_{W}^{(T)}(s)
$$

Finally we must decide at what value of $s$ we should evaluate $\hat{\Pi}_{W}^{(T)}(s)$. From our discussion at the end of the previous section, it is clear that it should make little difference, and that the best choice is that which corresponds to the most precise experiments. Since the best determinations of the electroweak couplings should come from experiments done at the electroweak boson resonances, the best choice is $s=M_{W}^{2}$. Thus, we finally obtain

$$
\delta g_{2}^{2}=-g_{2}^{2} \operatorname{Re} \hat{\Pi}_{W}^{(T)}\left(M_{W}^{2}\right)
$$

Similar expressions can be obtained for other couplings by considering other processes. From neutrino scattering one obtains

$$
\frac{\delta g_{1}^{2}+\delta g_{2}^{2}}{\left(g_{1}^{2}+g_{2}^{2}\right)}=-\operatorname{Re} \hat{\Pi}_{Z}^{(T)}\left(M_{Z}^{2}\right),
$$


while from quark scattering

$$
\delta g_{3}^{2}=-g_{3}^{2} \operatorname{Re} \hat{\Pi}_{g}^{(T)}\left(M_{Z}^{2}\right) .
$$

Here $^{2}$ In the $g_{1}$ and $g_{3}$ stand for the gauge couplings for the $U(1)$ and $S U(3)$ groups, respectively, and $\Pi_{Z}$ and $\Pi_{g}$ are the $Z$-boson and gluon self energies. Again, the hat over these quantities reminds us to include only non-standard model contributions.

These expressions for threshold corrections can be manipulated to express threshold corrections for derived quantities. Since this is often done, and in order to compare with existing results, we also cast our results in terms of the correction to the weak mixing angle,

$$
\delta \sin ^{2} \theta=\cos ^{2} \theta \operatorname{Re}\left[\hat{\Pi}_{W}^{(T)}\left(M_{W}^{2}\right)-\hat{\Pi}_{Z}^{(T)}\left(M_{Z}^{2}\right)\right]
$$

Here, the weak mixing angle is defined in terms of the gauge coupling constants, $\sin ^{2} \theta=$ $g_{1}^{2} /\left(g_{1}^{2}+g_{2}^{2}\right)$. Also, of specific interest to the SUSY-GUT theories is the light thresholds correction to the leading-log relation

$$
\sin ^{2} \theta=\frac{1}{5}+\frac{7}{15} \frac{\alpha_{\text {e.m. }}}{\alpha_{s}}
$$

Expressing this relation in terms of the couplings as extracted from experiment, that is, using the standard model couplings, one finds corrections to the relation,

$$
\sin ^{2} \theta=\frac{1}{5}+\frac{7}{15} \frac{\alpha_{\text {e.m. }}}{\alpha_{s}}+\delta_{\text {light }} .
$$

The correction $\delta_{\text {light }}$ was computed, using the run-and-match approach in ref. [7]. In section 7 we will compare the results of that and our calculation of $\delta_{\text {light }}$.

Using the results for $\delta g_{i}$ above, one obtains

$$
\delta_{\text {light }}=\frac{\alpha_{\text {e.m. }}}{20 \pi}\left[\frac{1}{\sin ^{4} \theta}\left(-1+2 \sin ^{2} \theta-5 \sin ^{4} \theta\right) \Phi_{W}+\frac{1}{\sin ^{4} \theta} \Phi_{Z}+\frac{7}{3} \Phi_{g}\right]
$$

where we have introduced the reduced self-energies

$$
\begin{aligned}
& \operatorname{Re} \hat{\Pi}_{W}^{(T)}\left(M_{W}^{2}\right)=\frac{\alpha_{\mathrm{e} . \mathrm{m}} \cdot}{4 \pi} \frac{1}{\sin ^{2} \theta} \Phi_{W} \\
& \operatorname{Re} \hat{\Pi}_{Z}^{(T)}\left(M_{Z}^{2}\right)=\frac{\alpha_{\mathrm{e} . \mathrm{m} .}}{4 \pi} \frac{1}{\sin ^{2} \theta \cos ^{2} \theta} \Phi_{Z} \\
& \operatorname{Re} \hat{\Pi}_{g}^{(T)}\left(M_{Z}^{2}\right)=\frac{\alpha_{s}}{4 \pi} \Phi_{g}
\end{aligned}
$$

2 In this section, the normalization of $g_{1}$ is the standard model one. In the following sections we will switch, without warning, to the normalization that is more appropriate for GUT theories. That is, we will absorb a factor of $\sqrt{5 / 3}$ in $g_{1}$. 
These are functions of the masses of the virtual particles. When any one such mass becomes large, the leading contribution to these functions is in the form of a logarithm of the ratio of the mass to the $M_{Z}$ or $M_{W}$. Thus one recovers the form of the result of the usual method, described earlier, of matching couplings and changing evolution equations at thresholds. We have checked that our expressions agree with the standard run-and-match formulas when we take the large mass, $S U(2)$ symmetric limit.

\section{Explicit Computations}

In the previous section we set up the stage for explicit computations. In this section we present explicit results of one-loop computations.

Lets consider first the case of scalar particles contributions to $\Phi_{X}$. The two required one-loop Feynman diagrams are shown in fig. 3. The calculation is performed using dimensional regularization and minimal subtraction. The result can be written succinctly in terms of the function

$$
I_{1}\left(m_{1}, m_{2}, q\right)=\frac{m_{1}^{2}}{q^{2}} \ln \frac{m_{1}^{2}}{\mu^{2}}+\frac{m_{2}^{2}}{q^{2}} \ln \frac{m_{2}^{2}}{\mu^{2}}-\frac{1}{3}-2 \int_{0}^{1} d x \frac{M^{2}(x)}{q^{2}} \ln \frac{M^{2}(x)}{\mu^{2}}
$$

where

$$
M^{2}(x) \equiv m_{2}^{2} x+m_{1}^{2}(1-x)-q^{2} x(1-x)
$$

and $\mu$ is the renormalization point. For a colored complex scalar of mass $m$ in the $R$ representation of color $S U(3)$, with Casimir invariant $C(R)$, one has

$$
\Phi_{g}=C(R) I_{1}\left(m, m, M_{Z}\right)
$$

The all important effect of mixing enters the computation of $\Phi_{Z}$ and $\Phi_{W}$. Writing the scalar field component of the neutral current as

$$
g_{i j} \phi_{i}^{*} \overleftrightarrow{\partial_{\mu}} \phi_{j}
$$

and the corresponding scalar masses as $m_{i, j}$, one obtains the $Z$ self-energy

$$
\Phi_{Z}=\left|g_{i j}\right|^{2} I_{1}\left(m_{i}, m_{j}, M_{Z}\right)
$$

The coupling $g_{i j}$ includes the usual group theoretic factors and gauge couplings, and the angles from the mixing matrix. Consider, for a definite example, a scalar of mass $m$ which 
does not mix, that is, for which the mass and weak eigenstates agree. We only need consider scalars in representations which are singlets or doublets under weak-SU(2). Let $\tau^{3}$ stand for the third component of weak-isospin and $Q$ for electric charge. One obtains,

$$
\Phi_{Z}=\left(\tau^{3}-\sin ^{2} \theta Q\right)^{2} I_{1}\left(m, m, M_{Z}\right)
$$

The expression for $\Phi_{W}$ is entirely analogous to that of $\Phi_{Z}$ in eqn. (4.3). Lets consider the case of no mixing. $\Phi_{W}=0$ for weak-singlets, while if the members of the doublet have masses $m_{1}$ and $m_{2}$

$$
\Phi_{W}=\frac{1}{2} I_{1}\left(m_{1}, m_{2}, M_{W}\right) .
$$

Next we turn to the case of fermions. There is only one Feynman diagram contribution to $\Phi_{X}$ at one-loop; see fig. 4 . In the case of fermions, three functions are needed to describe the results:

$$
\begin{aligned}
& I_{2}\left(m_{1}, m_{2}, q\right)=8 \int_{0}^{1} d x x(1-x) \ln M^{2}(x) / \mu^{2} \\
& I_{3}\left(m_{1}, m_{2}, q\right)=-\frac{4}{q^{2}} \int_{0}^{1} d x\left[m_{1}^{2} x+m_{2}^{2}(1-x)\right] \ln M^{2}(x) / \mu^{2} \\
& I_{4}\left(m_{1}, m_{2}, q\right)=\frac{4 m_{1} m_{2}}{q^{2}} \int_{0}^{1} d x \ln M^{2}(x) / \mu^{2}
\end{aligned}
$$

In terms of these, one has for colored fermions

$$
\Phi_{g}=C(R) I_{2}\left(m, m, M_{Z}\right)
$$

We will be generally concerned with right handed, as well as left handed, weak-doublets. Therefore it is useful to introduce some additional notation, allowing for the possibility of mixing in the neutral current:

$$
\frac{e}{2 \sin \theta \cos \theta} \bar{\psi}_{i}\left(g_{i j}^{L}\left(1-\gamma^{5}\right)+g_{i j}^{R}\left(1+\gamma^{5}\right)\right) \psi_{j}
$$

These couplings, $g^{L}$ and $g^{R}$, involve both mixing and the usual $\tau^{3}-\sin ^{2} \theta Q$ factors. In terms of these,

$$
\Phi_{Z}=\frac{1}{2}\left(\left|g_{i j}^{L}\right|^{2}+\left|g_{i j}^{R}\right|^{2}\right)\left(I_{2}\left(m_{i}, m_{j}, M_{Z}\right)+I_{3}\left(m_{i}, m_{j}, M_{Z}\right)\right)+\operatorname{Re}\left(g_{i j}^{L} g_{j i}^{R *}\right) I_{4}\left(m_{i}, m_{j}, M_{Z}\right)
$$


The expression for $\Phi_{W}$ can in fact be read off from this, but we give it explicitly for future reference. For a fermion doublet with components of mass $m_{1}$ and $m_{2}$,

$$
\Phi_{W}=\frac{1}{4}\left(I_{2}\left(m_{1}, m_{2}, M_{W}\right)+I_{3}\left(m_{1}, m_{2}, M_{W}\right)\right) .
$$

We have neglected mixing, which is a good approximation in the quark sector. Were we to neglect mixing in other sectors, this expression would be valid by replacing $1 / 4 \rightarrow$ $1 / 2 C$ (rep). In the case of the wino-zino multiplet, which transforms as the adjoint, the appropriate replacement is $1 / 4 \rightarrow 1$. But in addition one has to include the all important effect of mixing, and the corresponding formula is just as in eq. (4.10).

It should be kept in mind that the fermions in the neutralino sector are Majorana. The equations above apply to them too, provided one remembers to include factors of $1 / 2$ as appropriate.

Explicit expressions for the integrals appearing in eqs. (4.1) and (4.7) are easily written. We refrain from doing so because the expressions are lengthy. A detail discussion of the behavior of these functions can be found in section 7 below.

\section{Top and Higgs}

The experimental extraction of gauge couplings in the standard model depends on assumptions on two other undetermined parameters, the top and Higgs masses. It is customary to make a global fit of observables including not just the gauge couplings but these masses - or at least the top mass- as well. How are we to deal with this in our translation between standard and extended models?

Consider first the dependence on the Higgs mass. In supersymmetric models one must introduce two distinct Higgs doublets to ensure that all quarks and charged leptons are massive. Both fields get vacuum expectation values, and the physical spectrum is more complicated than that of the standard model. The details are spelled out in the next section. For now we would like to concentrate on the question of how to account for the different Higgs sectors in the standard model and the supersymmetric one. The problem is that the physical Higgs of the standard model generally has no simple direct correspondence in the supersymmetric extension. The linear combination of fields that gets a vacuum expectation value, a clear candidate for the corresponding standard model Higgs, is not a mass eigenstate. 
Thus, in computing $\delta g_{k}$ one must be careful to include these distinctions into $\Delta F_{i}$ in eq. (2.4). In fact, $\Delta F_{i}$ should include all the supersymmetric Higgs sector contributions and subtract the standard model ones. However, the standard model calculation introduces an unknown parameter, the Higgs mass. In fact, some of the uncertainty in the determination of the standard model's gauge couplings is due to the lack of determination of the Higgs mass. In our approach, the standard Higgs contribution would be replaced by a sum of contributions from the two neutral scalars, plus, in addition, genuinely new contributions. When the two neutral scalars are degenerate and with mass equal to that of the standard model's Higgs, this sum is exactly equal to the standard model Higgs contribution that it replaces. Because the mass of the Higgs is unknown, and since the standard model gauge couplings are not very sensitive to it, it is a good approximation to retain the standard model Higgs contribution, and instead to neglect the corresponding terms in the model with two Higgs doublets.

To summarize, given the uncertainties associated with the standard model determination of gauge couplings, the calculation of Higgs sector threshold corrections needs include only contributions from physical scalars in the $W$ and $Z$ self-energy diagrams of fig. 3 .

It must be noted here that in the run-and-match analysis this is accomplished by neglecting mixing between the two Higgs doublets. Then a correspondence is made between one of them and the standard model's, while the other gives a new light threshold correction.

The contribution from the top quark is identical in the standard model and its extension. It is included in the function $F_{i}\left(\tilde{g}_{k}\right)$ of eq. (2.4). The problem is that the determination of gauge couplings usually uses this as a fit variable. This could lead us into a complicated analysis of correlations. We are fortunate in that the models we are interested in

- supergravity unification with radiative-induced electroweak symmetry breaking - yield top quark masses in the range obtained from these fits. Therefore, to good approximation, the effect of top need not be included in our calculations.

\section{Review of the minimal $S U(5)$ supergravity model.}

In the next section we will illustrate our treatment of the light thresholds. In preparation we review here the minimal $S U(5)$ supergravity model. We also describe the procedure we have adopted for the calculation of the spectrum. The particle content of the model consist of three generation of quarks and leptons, the $S U(3) \times S U(2) \times U(1)_{Y}$ gauge bosons, 
two Higgs doublets and the supersymmetric particles of all these particles. We neglect the Yukawa couplings of the two light generations. The superpotential of the effective low energy theory thus has the form

$$
W=\lambda_{t} U^{c} Q h_{2}+\lambda_{b} D^{c} Q h_{1}+\lambda_{\tau} E^{c} L h_{1}+\mu h_{1} h_{2}
$$

where $Q$ and $L$ are quark and lepton doublet superfields, and $U^{c}, D^{c}$ and $E^{c}$ are the corresponding $S U(2)$ singlets; $h_{1}$ and $h_{2}$ are the two Higgs doublets. The soft supersymmetry breaking terms are

$$
\begin{aligned}
V_{\text {soft }}= & \left(\lambda_{t} A_{t} Q U^{c} h_{2}+\lambda_{b} A_{b} Q D^{c} h_{1}+\lambda_{\tau} A_{\tau} L E^{c} h_{1}+B \mu h_{1} h_{2}+\text { h.c. }\right)+ \\
& m_{h_{1}}^{2}\left|h_{1}\right|^{2}+m_{h_{2}}^{2}\left|h_{2}\right|^{2}+m_{L}^{2}|L|^{2}+m_{E^{c}}^{2}\left|E^{c}\right|^{2}+m_{Q}^{2}|Q|^{2}+m_{U^{c}}^{2}\left|U^{c}\right|^{2}+m_{D^{c}}^{2}\left|D^{c}\right|^{2} .
\end{aligned}
$$

The $S U(5)$ model is specified by the boundary conditions at the unification scale. The low energy parameters can then be determined from knowledge of their scale dependence. This is dictated by the renormalization group. The renormalization group equations (RGEs) for the minimal supersymmetric standard model are well known and are given in appendix A for completeness.

The boundary conditions at the unification scale that we adopt equate all of the trilinear coefficients $A_{X}$ and equate the hard scalar masses in the soft supersymmetry breaking potential of eqn. (6.2). In addition one has conditions that follow from minimal$S U(5)$ : equate the tau and beauty Yukawa couplings, $\lambda_{b}\left(M_{\mathrm{GUT}}\right)=\lambda_{\tau}\left(M_{\mathrm{GUT}}\right)$ and equate the gaugino masses $\tilde{M}_{i}(i=1,2,3$, corresponding to the $U(1) \times S U(2) \times S U(3)$ gauginos $)$. The free parameters are then the common trilinear coefficients $A$, the soft Higgs mass parameter $B$, the top Yukawa $\lambda_{t}$, the bilinear Higgs mixing coefficient $\mu$, the common gaugino mass $m_{\frac{1}{2}}$, the common scalar mass $m_{0}$, the scale of unification $M_{\mathrm{GUT}}$ and the unified gauge coupling $\alpha_{G U T}$. The free parameter $\lambda_{\tau}$ is of course fixed by the tau mass.

Given values of these free parameters one can compute the low energy spectrum of the theory, and then complete the calculation of the relation between gauge couplings at low energies as outlined in previous sections. We would like to explore the span of predictions that correspond to a large region of this free parameter space. It is quite unnecessary, though, to vary freely over values of all of these parameters. In computing the spectrum, we take as initial values $\sin ^{2} \theta_{W}=0.233$ and $\alpha_{s}=0.120$, from which we determine $M_{\mathrm{GUT}}$ and $\alpha_{G U T}$. 
The Yukawa couplings of the heavy generation are given by

$$
\lambda_{b, \tau}=\frac{1}{\cos \beta} \frac{m_{b, \tau}}{v} \quad \lambda_{t}=\frac{1}{\sin \beta} \frac{m_{t}}{v}
$$

where $v=\sqrt{v_{1}^{2}+v_{2}^{2}}=246 / \sqrt{2} \mathrm{GeV}, \tan \beta=v_{2} / v_{1}$ and $v_{1,2}$ refer to the expectation values of the two Higgs doublets. We obtain the heavy generation Yukawa couplings by eq. (6.3) from the input parameters $m_{t}$ and $\tan \beta$, and the input values of $m_{\tau}=1.78 \mathrm{GeV}$ and $m_{b}=4.0 \mathrm{GeV}$ at the $Z$-scale. In our numerical analysis, we evolve the Yukawa couplings from the $Z$-scale to $M_{\mathrm{GUT}}$, and constrain the parameter space by the requirement $\lambda_{b}\left(M_{\mathrm{GUT}}\right)$ differs from $\lambda_{\tau}\left(M_{\mathrm{GUT}}\right)$ by no more than $5 \%$. In so doing we neglect the effect of thresholds on the running of these Yukawa couplings. Thus for this procedure we need also a priori knowledge of the angle $\beta$.

In the next step in our numerical computation of the spectrum we evolve the Yukawa couplings, the soft SUSY breaking parameters and the gauge couplings with the boundary conditions specified at $M_{\mathrm{GUT}}$, to the electroweak scale, with the RGEs of the MSSM as given in appendix $\mathrm{A}$. We then obtain the supersymmetric spectrum at the weak scale from the equations given below.

In order to span over parameter space at the scale of grand-unification, and compute the low energy spectrum via the RGE's subject to the conditions that we obtain the correct masses of the tau-lepton and bottom-quark and that the correct electroweak breaking scale is the minimum of the tree level neutral Higgs potential, we trade the input parameters $\mu$ and $B$ for $v$ and $\tan \beta$ (or, equivalently, $v_{1}$ and $v_{2}$ ). Therefore, $B$ and $\mu$ become computed parameters. This we can do because the running of the SUSY parameters, as given by the RGE's in appendix A, does not depend on the values of $B$ or $\mu$ (except for $\mu$ itself). Thus, in our numerical analysis we span over the space generated by $A, m_{t}, m_{\frac{1}{2}}, m_{0}$ and $\tan \beta$, and compute the spectrum for each set of values.

We turn now to a description of the spectrum itself. We lay down here the basis for our numerical treatment of the next section, making no attempt to describe the spectrum's salient qualitative features. A nice account of this can be found in ref. [12] while extensive numerical analysis of the spectrum can be found in ref. [13]. Our results for the sparticle spectrum agree qualitatively with the results of these references. For the two light generation sparticles we neglect the Yukawa couplings in the RGE's. The light-generation sparticle masses may then be analytically calculated from the one-loop RGEs in terms of the three unknowns $m_{\frac{1}{2}}, m_{0}$, and $\cos 2 \beta$ :

$$
m_{\tilde{p}}^{2}=m_{0}^{2}+c_{\tilde{p}} m_{\frac{1}{2}}^{2}+d_{\tilde{p}}
$$


The coefficients $c_{\tilde{p}}$ for the different sparticles are

$$
c_{\tilde{p}}=c_{3}\left(m_{\tilde{p}}\right)+c_{2}\left(m_{\tilde{p}}\right)+Y_{\tilde{p}}^{2} c_{y}\left(m_{\tilde{p}}\right)
$$

with

$$
\begin{aligned}
& c_{3}\left(m_{\tilde{p}}\right)=-\frac{8}{9}\left(1-\left(1+\frac{3 \alpha_{G U T} t}{2 \pi}\right)^{-2}\right) \\
& c_{2}\left(m_{\tilde{p}}\right)=\frac{3}{2}\left(1-\left(1-\frac{\alpha_{G U T t}}{2 \pi}\right)^{-2}\right) \\
& c_{y}\left(m_{\tilde{p}}\right)=\frac{10}{33}\left(1-\left(1-\frac{33 \alpha_{G U T t} t}{10 \pi}\right)^{-2}\right)
\end{aligned}
$$

where $\alpha_{G U T}$ is the coupling at the unification scale and $t=\log \left(m_{\tilde{p}} / M_{\mathrm{GUT}}\right)$. The RGE's are integrated to the physical sparticle mass to obtain the $c$ 's. The $d$ 's arise from D-terms in the potential and are given by

$$
d_{\tilde{p}}=2\left(T_{3_{L}}^{\tilde{p}}-\frac{3}{5} Y^{\tilde{p}} \tan ^{2} \theta_{W}\right) \cos 2 \beta M_{W}^{2} .
$$

In case of small $\tan \beta$ the bottom quark and tau lepton Yukawa couplings can be neglected as well. In the case of large $\tan \beta$ the heavy generation sparticle mass matrices are given by

$$
\begin{aligned}
& M_{\tilde{t}}^{2}=\left(\begin{array}{cc}
m_{Q}^{2}+m_{t}^{2}+d_{\tilde{u}_{l}} & m_{t}\left(A_{t}+\mu \cot \beta\right) \\
m_{t}\left(A_{t}+\mu \cot \beta\right) & m_{U^{c}}^{2}+m_{t}^{2}+d_{\tilde{u}_{r}}
\end{array}\right) \\
& M_{\tilde{b}}^{2}=\left(\begin{array}{cc}
m_{Q}^{2}+m_{b}^{2}+d_{\tilde{d}_{l}} & m_{b}\left(A_{b}+\mu \tan \beta\right) \\
m_{b}\left(A_{b}+\mu \tan \beta\right) & m_{D^{c}}^{2}+m_{b}^{2}+d_{\tilde{d}_{r}}
\end{array}\right) \\
& M_{\tilde{\tau}}^{2}=\left(\begin{array}{cc}
m_{L}^{2}+m_{\tau}^{2}+d_{\tilde{e}_{l}} & m_{\tau}\left(A_{\tau}+\mu \tan \beta\right) \\
m_{\tau}\left(A_{\tau}+\mu \tan \beta\right) & m_{E^{c}}^{2}+m_{\tau}^{2}+d_{\tilde{e}_{r}}
\end{array}\right)
\end{aligned}
$$

The mass eigenstates are obtained by diagonalizing the mass matrices above by a $2 \times 2$ unitary transformation.

The chargino and neutralino mass matrices are given by

$$
M_{\tilde{C}}=\left(\begin{array}{cc}
\tilde{M}_{2} & M_{W} \sqrt{2} \sin \beta \\
M_{W} \sqrt{2} \cos \beta & \mu
\end{array}\right),
$$

and

$$
M_{\tilde{N}}=\left(\begin{array}{cccc}
\tilde{M}_{1} & 0 & -M_{Z} \sin \theta_{W} \cos \beta & M_{Z} \sin \theta_{W} \sin \beta \\
0 & \tilde{M}_{2} & M_{Z} \cos \theta_{W} \sin \beta & -M_{Z} \cos \theta_{W} \cos \beta \\
-M_{Z} \sin \theta_{W} \cos \beta & M_{Z} \cos \theta_{W} \sin \beta & 0 & \mu \\
M_{Z} \sin \theta_{W} \sin \beta & -M_{Z} \cos \theta_{W} \cos \beta & \mu & 0
\end{array}\right)
$$


respectively, where $\tilde{M}_{i}=\left(\alpha_{i} / \alpha_{U}\right) m_{\frac{1}{2}}(i=1,2,3)$ are the gaugino masses at their mass scale. The mass eigenstates and mixing can be found in ref. [14].

There are two complex Higgs doublets in the MSSM, $\left(h_{2}^{+}, h_{2}^{0}\right)$, whose neutral vacuum expectation value (VEV) $v_{2}$ gives mass to the $\frac{2}{3}$ charged quarks, and $\left(h_{1}^{0}, h_{1}^{-}\right)$whose neutral VEV gives mass to the $-\frac{1}{3}$ charged quarks. Of the eight real degrees of freedom, three are the Goldstone bosons that are absorbed by the standard model gauge bosons. The CPodd neutral Higgs boson $A^{0}$ and the orthogonal combinations $h^{+}=\sin \beta\left(h_{1}^{-}\right)^{*}+\cos \beta h_{2}^{+}$, $h^{-}=\left(h^{+}\right)^{*}$ correspond to physical particles with masses

$$
m_{A}^{2}=-m_{3}^{2}(\tan \beta+\cot \beta)
$$

and

$$
m_{h \pm}^{2}=m_{W}^{2}+m_{A}^{2},
$$

where $m_{3}=B \mu$ The two CP-even neutral Higgs bosons mass eigenstates are obtained by diagonalizing

$$
M_{R}^{2}=\frac{\sin 2 \beta}{2}\left(\begin{array}{cc}
\cot \beta M_{Z}^{2}+\tan \beta m_{A}^{2} & -\left(M_{Z}^{2}+m_{A}^{2}\right) \\
-\left(M_{Z}^{2}+m_{A}^{2}\right) & \tan \beta M_{Z}^{2}+\cot \beta m_{a}^{2}+\Delta
\end{array}\right),
$$

where $\Delta$ is the one loop radiative correction to the neutral Higgs boson masses[15]

$$
\Delta=\frac{3}{8 \pi^{2}} \frac{g^{2} m_{t}^{4} \cot \beta}{m_{W}^{2}} \log \left(1+\frac{m_{s q}^{2}}{m_{t}^{2}}\right) .
$$

The linear combination of Higgs fields that gets a VEV, $h_{s m}$, and the one that does not get a VEV, $h_{s u s y}$, are obtained from the mass eigenstates $h$ and $H$ by a rotation,

$$
\left(\begin{array}{c}
h_{s m} \\
h_{s u s y}
\end{array}\right)=\left(\begin{array}{cc}
\cos (\alpha-\beta) & \sin (\alpha-\beta) \\
-\sin (\alpha-\beta) & \cos (\alpha-\beta)
\end{array}\right)\left(\begin{array}{c}
h \\
H
\end{array}\right)
$$

where $\alpha$ is the angle that rotates from the gauge to the mass eigenstates. Requiring a negative eigenvalue for the neutral Higgs mass-squared matrix and that the Higgs potential is bounded from below imposes two conditions on the running of the mass parameters:

$$
\begin{aligned}
& \text { 1. } m_{1}^{2} m_{2}^{2}-m_{3}^{4}<0 \\
& \text { 2. } m_{1}^{2}+m_{2}^{2}-2\left|m_{3}^{2}\right|>0
\end{aligned}
$$

where $m_{1,2}^{2}=m_{h_{1,2}}^{2}+\mu^{2}$ and $m_{3}^{2}=B \mu$. 


\section{Numerical Results and Discussion}

We first consider the functions $\Phi_{X}$ of section 4 as compared with the naive (leading$\log$, or "run-and-match") result. The latter can be obtained by the standard run-andmatch computation, or simply by taking the large $m / M_{Z}$ limit of our expressions. Both procedures must agree when in our expressions $S U(2) \times U(1)$ breaking is neglected.

Consider the contributions of scalars to $\delta_{\text {light }}$ of eq. (3.14), both in our procedure and using the run-and-match approximation. When $S U(2) \times U(1)$ breaking is neglected, so that all members of a weak-isospin multiplet are degenerate, all three functions $\Phi_{X}$ are simply proportional to the function $I_{1}$. Thus, for the contribution of any one scalar, the ratio of $\delta_{\text {light }}$ computed in the run-and-match approximation to our result is simply the ratio of the leading-log term in $I_{1}$ to the whole expression for $I_{1}$. This ratio is plotted in fig. 5 as a function of $m / M_{Z}$. It is apparent that the error in the approximation is small except for $m / M_{Z} \approx 1$.

Next, consider the contributions of fermions to $\delta_{\text {light }}$. The $\Phi_{g}$ piece is easiest to analyze, since it is simply proportional to the function $I_{2}$; see eqs. (4.7a) and (4.8). In fig. 6 we plot the ratio of the naive run-and-match approximation to the complete one loop threshold correction function $\Phi_{g}$, due to virtual fermions. In this case, again, the convergence to the naive run-and-match limit is extremely fast.

The most interesting case is that of the fermion contributions to $\Phi_{Z}$ and $\Phi_{W}$. Recall, from eq. (4.10) that $\Phi_{Z}$ is a linear combinations of the integrals $I_{2}, I_{3}$ and $I_{4}$, and $\Phi_{W}$ of $I_{2}$ and $I_{3}$. The behavior of $I_{2}$ was analyzed above, and was found to be quite uninteresting. Turning our attention to $I_{3}$, we see by inspection of eq. (4.7b) that its asymptotic behavior, for large $m$, is $I_{3}(m, m, q) \sim-4 m^{2} / q^{2} \ln \left(m^{2} / \mu^{2}\right)+2 / 3+q^{2} / 15 m^{2}+\mathcal{O}\left(q^{4} / m^{4}\right)$. This is an example of non-decoupling, in which the leading term grows as a power (rather than a $\log$ ) of the mass. In fact, the combination $\Phi_{Z}-\Phi_{W}$ is precisely what enters the computation of Veltman's $\rho$ parameter, which is well known to grow as $\sim m_{t}^{2}$ in the standard model[16]. Notice though that for particles in vectorial representations the prefactor $\left(\tau_{L}^{3}-\tau_{R}^{3}\right)^{2}$ vanishes. So a large effect will depend on the breaking of $S U(2) \times U(1)$.

It is interesting to note that the combination $I_{3}+I_{4}$ which often enters into $\Phi_{Z}$, vanishes in the limit of degenerate multiplets. This can be seen by writing it as

$$
I_{3}\left(m_{1}, m_{2}, q\right)+I_{4}\left(m_{1}, m_{2}, q\right)=\left(m_{1}-m_{2}\right) \int_{0}^{1} d x\left(x m_{1}-(1-x) m_{2}\right) \log M^{2}(x) / \mu^{2} .
$$


These type of leading corrections to $\delta_{\text {light }}$ are missed altogether by the naive runand-match calculation. In fact, there is no pure $\log$ term in $I_{3}$, an indication that it is invisible to the naive approach. And, clearly, the combination $I_{3}+I_{4}$ is missed altogether too. Therefore we cannot quantify the effects of these terms, relative the run-and-match approach, in a manner similar to the above examples. We postpone further consideration until we come to computations in the full $S U(5)$ supergravity model, below.

To get some idea of how important the $S U(2)$ breaking effects can be, we can look at the contribution to $\delta_{\text {light }}$ from a doublet of scalars, with masses $m_{1}$ and $m_{2}$ respectively, and compare to the would be contribution from a degenerate doublet with some average mass, $m_{\text {avg }}$. For definiteness we shall take $m_{\text {avg }}=\left(m_{1}+m_{2}\right) / 2$, although one could just as well use, say, $m_{\text {avg }}=\sqrt{m_{1} m_{2}}$. In fig. 7 we plot the ratio $I_{1}\left(m_{1}, m_{2}, q\right) / I_{1}\left(m_{\text {avg }}, m_{\text {avg }}, q\right)$ for $m_{2} / q=1.5,3.5,5.5$ and 7.5 (corresponding to the ever increasing location of the peaks in the graph). It is clear that $S U(2)$ breaking effect can be rather considerable. It should be pointed out that large $S U(2)$ breaking effects would show up as significant deviations from unity of Veltman's $\rho$-parameter. For consistency with the observed value of $\rho$, the parameters of the SUSY-GUT are such that the $S U(2)$ breaking effects are small.

These comparisons, although instructive, are incomplete. They miss the possibility of cumulative effects of the many particles in the rich supersymmetric spectrum. We therefore use our equations in the context of the model described in the previous section. This will allow consideration of the effects of mixing. These are obviously important and altogether missed by the run-and-match approach, but serious consideration of them requires a choice of reasonable mixings. In the minimal supergravity model based on $S U(5)$ the mixing is completely fixed in terms of other more fundamental parameters.

We calculate the spectrum in the minimal supersymmetric $S U(5)$ model as described in section 6. There are five input parameters, namely $A, m_{0}, m_{\frac{1}{2}}, \tan \beta$ and the top quark mass $m_{t}$. They are constrained by the requirement of radiatively induced symmetry breaking of the electroweak group, and lower bounds on supersymmetric particle masses. We perform a systematic, although rather coarse-grained, search through parameter space. Each of the five parameters is allowed to vary over a reasonable range. The range is sampled at some fixed predetermined interval size. Table 1 summarizes the range and size of the sampling interval for each of the five parameters. This represents a sampling of more than $4 \times 10^{4}$ points. Of the resulting spectrum we require that it does not violate experimental bounds: we require that chargino, squark, gluino, slepton and neutralino masses to be in excess of $45 \mathrm{GeV}, 100 \mathrm{GeV}, 150 \mathrm{GeV}, 43 \mathrm{GeV}$ and $20 \mathrm{GeV}$, respectively. We also require 
that the lightest neutralino is lighter than the chargino. Only some $1.5 \times 10^{4}$ sampling points in parameter space survive this requirements. To study the dependence on each of the input parameters we use a finer grind for one of the inputs and take two or three extreme points for the other input parameters. We find that $m_{t}<160 \mathrm{GeV}$ and $\tan \beta<30$. This is the case in part because we have assumed that the tau and bottom Yukawa couplings are equal at the unification scale. The gluino mass limit, $M_{\tilde{g}}>150 \mathrm{GeV}$, constrains $m_{\frac{1}{2}}$ to be above $55 \mathrm{GeV}$. Upper limits on $\left|A_{0}\right|, m_{0}$ and $m_{\frac{1}{2}}$ can be set by resorting to naturalness arguments [5]. However, as our interest is in the SUSY spectrum close to the electroweak scale, we impose upper bounds on these parameters not far above the electroweak scale.

\begin{tabular}{|c|r|r|r|}
\hline & \multicolumn{1}{|c|}{$X_{i}$} & $X_{f}$ & $\Delta X$ \\
\hline$A_{0}$ & -200 & 200 & 50 \\
$m_{0}$ & 0 & 400 & 50 \\
$m_{12}$ & 100 & 300 & 25 \\
$\tan \beta$ & 2 & 30 & 4 \\
$m_{\boldsymbol{t}}$ & 110 & 170 & 10 \\
\hline
\end{tabular}

Table 1. The range and sampling size of the parameter space. Each free parameter $X$ is sampled in the interval $\left(X_{i}, X_{f}\right)$ with spacing $\Delta X$ between consecutive points.

How good is the run-and-match analysis in this case? In fig. 8 we compare our calculation with the result of ref. [7]. We plot $\sin ^{2} \theta$, calculated in (3.13) using $\alpha_{s}=0.120$ and $\alpha_{\text {e.m. }}=1 / 127.9$, against $\sin ^{2} \theta$ in the run-and-match approximation. Each point on this plot represents a specific choice of the free parameters. It is clear that for many points the run-and-match approximation is far too crude to be useful. In fact, we find that $92 \%$ of the points in parameter space give $\delta_{\text {light }}$ which differs from the run-and-match approximation by more than $30 \%$. 
In figs. 9--12 we plot the dependence of $\sin ^{2} \theta$ in our calculation on the mass of a few of the superparticles. The dependence on the lightest chargino mass, $M_{C, \min }$, can be seen in fig. 9. As expected, the points tend to concentrate around the $\sin ^{2} \theta$ value of the run-and-match approximation as $M_{C, \min }$ increases. The mass of the lightest neutral Higgs increases rapidly as a function of $\tan \beta$, reaching a limiting value of about $100 \mathrm{GeV}$ at $\tan \beta \sim 6$. This is the reason for the two band structure of fig. 10, a scatter plot of $\sin ^{2} \theta$ against $M_{H, \min }$. The band at about $60 \mathrm{GeV}$ is made up mostly of points at $\tan \beta=2$, while the band at $100 \mathrm{GeV}$ consists mostly of points with $\tan \beta \geq 6$. Fig. 11 shows $\sin ^{2} \theta$ against the mass of the lighter of the two top-squarks. It is similar to fig. 9 in that points tend to accumulate at the run-and-match value as $M_{T, \min }$ increases, although the spread seems larger. Also worth noticing is the rapid monotonic variation at low values of $M_{T, \min }$. This is not surprising, since, as we have argued repeatedly, the effects of our calculation should be more noticeable for lighter than for heavier spectra. Fig. 12 again demonstrates this effects, now as a function of the lightest neutralino.

We would like to emphasize that our numerical analysis is not intended as a complete analysis of the parameter space and of the SUSY spectrum. Nor do we believe, in view of the huge parameter space, that such analysis carry substantial meaning. The parameter space can be constrained by making specific assumptions, motivated to some extent by theoretical considerations, on the form of the Kaeler potential in the supergravity model, and consequently on the initial boundary conditions at the unification scale. Partial analysis of the SUSY spectrum, based on such specific assumptions, have been performed by several groups[13]. In this paper, our purpose is to address the question of how to appropriately account for the effects of light thresholds, and to illustrate the need for a proper method to do so. Our numerical analysis should be regarded merely as illustrative.

\section{Conclusions}

The standard run-and-match approach to the calculation of light threshold effects is seen to be very inaccurate when the supersymmetric spectrum is not too heavy and there is substantial $S U(2)$ breaking and mixing. We have described a better approximation which incorporates these effects. Although we have concentrated on the predicted value of $\sin ^{2} \theta$ in the numerical analysis in this work, clearly similar analysis apply to the calculation of other quantities of interest. For example, one may include threshold effects in the calculation of the spectrum itself[17]. 
The calculation that we have presented is a sensible approach, while supersymmetric particles are not found and provided there is no evidence of experimental deviations from standard model expectations. When either of these assumptions is violated the calculation should be replaced by a full calculation of the novel process, including possible non-universal effects, that is, non-oblique corrections.

In the mean time one may use this type of calculation to restrain more severely the allowed parameter space of supersymmetric grand-unified theories. It would be interesting to produce an analysis including simultaneously the predictions of $\sin ^{2} \theta$ and other restricting quantities, to better limit the boundaries of parameters space. For example, the inclusive rate for radiative $B$ decays may be fairly restrictive of extreme values of $\tan \beta$. Unfortunately, to the extent that the standard model is successful, one can always find a region of the SUSY-GUT that satisfies all experimental constraints, for the sparticles decouple as they become much heavier than the massive vector bosons, and in that limit the predicted value of $\sin ^{2} \theta$ is consistent with experiment.

\section{Acknowledgments}

One of us (BG) would like to thank the Alfred P. Sloan Foundation for partial support. AF would like to thank the Feinberg school for partial support and the SSC laboratory for its hospitality while part of this work was conducted. This work is supported in part by the Department of Energy under contract DE-AC35-89ER40486.

\section{Appendix A. RGE's for the MSSM}

The renormalization group equation for the minimal supersymmetric standard model are well known and are given here for completeness.

$$
\begin{aligned}
\frac{d \lambda_{t}}{d t} & =\frac{\lambda_{t}}{8 \pi^{2}}\left(3 \lambda_{t}^{2}+\frac{1}{2} \lambda_{b}^{2}-\frac{8}{3} g_{3}^{2}-\frac{3}{2} g_{2}^{2}-\frac{13}{30} g_{1}^{2}\right) \\
\frac{d \lambda_{b}}{d t} & =\frac{\lambda_{b}}{8 \pi^{2}}\left(3 \lambda_{b}^{2}+\frac{1}{2} \lambda_{t}^{2}+\frac{1}{2} \lambda_{\tau}^{2}-\frac{8}{3} g_{3}^{2}-\frac{3}{2} g_{2}^{2}-\frac{7}{30} g_{1}^{2}\right)
\end{aligned}
$$




$$
\begin{aligned}
\frac{d \lambda_{\tau}}{d t} & =\frac{\lambda_{\tau}}{8 \pi^{2}}\left(2 \lambda_{\tau}^{2}+\frac{3}{2} \lambda_{b}^{2}-\frac{3}{2} g_{2}^{2}-\frac{9}{10} g_{1}^{2}\right) \\
\frac{d \mu}{d t} & =\frac{\mu}{8 \pi^{2}}\left(\frac{3}{2} \lambda_{t}^{2}+\frac{3}{2} \lambda_{b}^{2}+\frac{1}{2} \lambda_{\tau}^{2}-\frac{3}{2} g_{2}^{2}-\frac{9}{10} g_{1}^{2}\right) \\
\frac{d A_{t}}{d t} & =\frac{1}{4 \pi^{2}}\left(3 \lambda_{t}^{2} A_{t}+\frac{1}{2} \lambda_{b}^{2} A_{b}+\frac{8}{3} g_{3}^{2} \tilde{M}_{3}+\frac{3}{2} g_{2}^{2} \tilde{M}_{2}+\frac{13}{30} g_{1}^{2} \tilde{M}_{1}\right) \\
\frac{d A_{b}}{d t} & =\frac{1}{4 \pi^{2}}\left(3 \lambda_{b}^{2} A_{b}+\frac{1}{2} \lambda_{t}^{2} A_{t}+\frac{1}{2} \lambda_{\tau}^{2} A_{\tau}+\frac{8}{3} g_{3}^{2} \tilde{M}_{3}+\frac{3}{2} g_{2}^{2} \tilde{M}_{2}+\frac{7}{30} g_{1}^{2} \tilde{M}_{1}\right) \\
\frac{d A_{\tau}}{d t} & =\frac{1}{4 \pi^{2}}\left(2 \lambda_{\tau}^{2} A_{\tau}+\frac{3}{2} \lambda_{b}^{2} A_{b}+\frac{8}{3} g_{3}^{2} \tilde{M}_{3}+\frac{3}{2} g_{2}^{2} \tilde{M}_{2}+\frac{9}{10} g_{1}^{2} \tilde{M}_{1}\right) \\
\frac{d B}{d t} & =\frac{1}{4 \pi^{2}}\left(\frac{3}{2} \lambda_{t}^{2} A_{t}+\frac{3}{2} \lambda_{b}^{2} A_{b}+\frac{1}{2} \lambda_{\tau}^{2} A_{\tau}+\frac{3}{2} g_{2}^{2} \tilde{M}_{2}+\frac{3}{10} g_{1}^{2} \tilde{M}_{1}\right) \\
\frac{d m_{h_{1}}^{2}}{d t} & =\frac{1}{8 \pi^{2}}\left(3 F_{b}+F_{\tau}-3 g_{2}^{2} \tilde{M}_{2}^{2}+\frac{3}{5} g_{1}^{2} \tilde{M}_{1}^{2}\right) \\
\frac{d m_{h_{2}}^{2}}{d t} & =\frac{1}{8 \pi^{2}}\left(3 F_{t}-3 g_{2}^{2} \tilde{M}_{2}^{2}+\frac{3}{5} g_{1}^{2} \tilde{M}_{1}^{2}\right) \\
\frac{d \tilde{M}_{Q}^{2}}{d t} & =\frac{1}{8 \pi^{2}}\left(F_{b}+F_{t}-\frac{16}{3} g_{3}^{2} \tilde{M}_{3}^{2}-3 g_{2}^{2} \tilde{M}_{2}^{2}-\frac{1}{15} g_{1}^{2} \tilde{M}_{1}^{2}\right) \\
\frac{d m_{U^{c}}^{2}}{d t} & =\frac{1}{8 \pi^{2}}\left(2 F_{t}-\frac{16}{3} g_{3}^{2} \tilde{M}_{3}^{2}-\frac{16}{15} g_{1}^{2} \tilde{M}_{1}^{2}\right) \\
\frac{d m_{D^{c}}^{2}}{d t} & =\frac{1}{8 \pi^{2}}\left(2 F_{b}-\frac{16}{3} g_{3}^{2} \tilde{M}_{3}^{2}-\frac{4}{15} g_{1}^{2} \tilde{M}_{1}^{2}\right) \\
\frac{d m_{L}^{2}}{d t} & =\frac{1}{8 \pi^{2}}\left(F_{\tau}-3 g_{2}^{2} \tilde{M}_{2}^{2}-\frac{3}{5} g_{1}^{2} \tilde{M}_{1}^{2}\right) \\
\frac{d m_{E^{c}}^{2}}{d t} & =\frac{1}{8 \pi^{2}}\left(2 F_{\tau}-\frac{6}{5} g_{1}^{2} \tilde{M}_{1}^{2}\right)
\end{aligned}
$$

where,

$$
\begin{aligned}
& F_{t}=\lambda_{t}^{2}\left(m_{Q}^{2}+m_{U^{c}}^{2}+m_{h_{2}}^{2}+A_{t}^{2}\right) \\
& F_{b}=\lambda_{b}^{2}\left(m_{Q}^{2}+m_{U^{c}}^{2}+m_{h_{1}}^{2}+A_{b}^{2}\right) \\
& F_{\tau}=\lambda_{\tau}^{2}\left(m_{L}^{2}+m_{E^{c}}^{2}+m_{h_{1}}^{2}+A_{\tau}^{2}\right)
\end{aligned}
$$




\section{References}

[1] The ALEPH collaboration, Phys. Lett. B284 (1992) 163;

The L3 collaboration, Phys. Lett. B284 (1992) 471;

The DELPHI collaboration, Z. Phys. C 54 (1992) 55;

The OPAL collaboration, Z. Phys. C 55 (1992) 1;

The LEP collaborations, ALEPH, DELPHI, L3, OPAL, Phys. Lett. B276 (1992) 247;

The ALEPH collaboration, Z. Phys. C 53 (1992) 1.

[2] U. Amaldi et al, Phys. Rev, D36 (1987) 1385;

P. Langacker, Proc. PASCOS-90 Symposium, Eds. P. Nath and S. Reucroft (World Scientific, Singapore, 1990);

P. Langacker and M. Luo, Phys. Rev. D44 (1991) 817;

J. Ellis, S. Kelley and D. V. Nanopoulos, Phys. Lett. B249 (1990) 441;

Phys. Lett. B260 (1991) 131;

U. Amaldi, W. de Boer and H. Füstenau, Phys. Lett. B260 (1991) 447;

H. Arason et al, Phys. Rev. D46 (1992) 3945;

F. Anselmo, L. Cifarelli, A. Peterman and A. Zichichi, Nuovo Cimento 105A (1992) 1179.

[3] R. Barbieri and L.J. Hall, Phys. Rev. Lett. 68 (1992) 752.

[4] A. E. Faraggi, B. Grinstein and S. Meshkov, Phys. Rev. D47 (1993) 5018;

K. Hagiwara and Y. Yamada, Phys. Rev. Lett. 70 (1993) 709.

[5] G. G. Ross and R. G. Roberts, Nucl. Phys. B377 (1992) 571.

[6] P. Langacker and N. Polonski, Phys. Rev. D47 (1993) 4028.

[7] J. Ellis, S. Kelley and D. V. Nanopoulos, Nucl. Phys. B373 (1992) 55; Phys. Lett. B287 (1992) 95.

[8] G. Passarino and M. Veltman, Nucl. Phys. B160 (1979) 151;

A. Sirlin, Phys. Rev. D22 (1980) 285;

A. Sirlin and W. J. Marciano, Nucl. Phys. B189 (1981) 442;

M. Consoli, Nucl. Phys. B160 (1979) 208;

F. Antonelli, M. Consoli and G. Corbo, Phys. Lett. 91B (1980) 90;

M. Veltman, Phys. Lett. $91 B$ (1980) 95;

M. Lemoine and M. Veltman, Nucl. Phys. B164 (1980) 445;

M. Green and M. Veltman, Nucl. Phys. B169 (1980) 137; erratum ibid, B175 (1980) 547 ;

M. Bohm and W. Hollik, Nucl. Phys. B204 (1982) 45; idem, Phys. Lett. 139B (1984) 213;

M. B. Einhorn, D.R.T. Jones and M. Veltman, Nucl. Phys. B191 (1981) 146;

W. J. Marciano and A. Sirlin, Phys. Rev. D27 (1983) 552; ibid, D29 (1984) 75; ibid 
p.945; erratum, ibid, D31 (1985) 213;

W. J. Marciano and A. Sirlin, in Brookhaven 1981, Proceedings, Isabelle, Vol. 1, 289302, 1981;

B. W. Lynn, M. E. Peskin and R. G. Stuart, in Physics at LEP, Vol. 1, J. Ellis and R. Peccei, eds., CERN 86-02, February 1986;

W. Hollik, Z. Phys. C37 (1988) 569;

D. Kennedy and B.W. Lynn, Nucl. Phys. B322 (1989) 1.

[9] B. W. Lynn, SLAC-PUB-3358, June 1984 (unpublished);

K. H. G. Schwarzer, OXFORD-TP 40/84, September 1984 (unpublished).

[10] B. W. Lynn, in Polarization at LEP, Vol. 1, G. Alexander et al, eds., CERN 88-06, September 1988

[11] A. Giveon, L.J. Hall and U. Sarid, Phys. Lett. B271 (1991) 138.

[12] S. Martin and P. Ramond, Sparticle Spectrum Constraints, NUB-3067-93TH, UFIFTHEP-93-16, SSCL-Preprint-439, June 1993 (unpublished).

[13] J. Ellis and F. Zwirner, Nucl. Phys. B338 (1990) 317;

S. Kelley, J. Lopez, H. Pois, D.V. Nanopoulos and K. Yuan, Phys. Lett. B273 (1991) 423 ;

M. Drees and M.M. Nojiri, Nucl. Phys. B369 (1992) 54;

P. Nath and R. Arnowitt, Phys. Rev. Lett. 69 (1992) 725.

[14] B. Grinstein, J. Polchinski and M. B. Wise, Phys. Lett. B130 (1983) 285

[15] Y. Okada, M. Yamaguchi and T. Yanagida, Prog. Theor. Phys. Lett. 85 (1991) 1;

J. Ellis, G. Ridolfi and F. Zwirner, Phys. Lett. B257 (1991) 83;

H. E. Haber and R. Hempfling, Phys. Rev. Lett. 66 (1991) 1815.

[16] M. Veltman, Nucl. Phys. B123 (1977) 89;

M.S. Chanowitz, M.A. Furman and I. Hinchliffe, Phys. Lett. B78 (1978) 285 and Nucl. Phys. B153 (1979) 402.

[17] L.J. Hall, R. Rattazzi and U. Sarid, The Top Quark Mass in Supersymmetric SO(10) Unification, LBL-33997, UCB-PTH-93/15, hep-ph/9306309 (unpublished). 


\section{Figure Captions}

Fig. 1. Tree level Feynman diagram for the scattering amplitude for $\mu \bar{\nu}_{\mu} \rightarrow e \bar{\nu}_{e}$ scattering.

Fig. 2. Feynman diagrams for one loop virtual effects of new heavy particles.

Fig. 3. One-loop Feynman diagrams in the calculation of threshold corrections: scalar particle contributions.

Fig. 4. One-loop Feynman diagram in the calculation of threshold corrections: fermion contributions.

Fig. 5. Ratio of the naive run-and-match approximation to the complete one loop threshold correction functions $\Phi_{X}$, due to a virtual scalar.

Fig. 6. Ratio of the naive run-and-match approximation to the complete one loop threshold correction function $\Phi_{g}$, due to virtual fermions.

Fig. 7. The effect of $S U(2)$ breaking, as included in our analysis, in the scalar contribution to $\Phi_{W}$. This is a plot of the ratio $I_{1}\left(m_{1}, m_{2}, q\right) / I_{1}\left(m_{\text {avg }}, m_{\text {avg }}, q\right)$, where $m_{\text {avg }}=\left(m_{1}+m_{2}\right) / 2$, for $m_{2} / q=1.5,3.5,5.5$ and 7.5 (corresponding to the ever increasing location of the peaks in the graph).

Fig. 8. Plot of the calculated $\sin ^{2} \theta$, this work, versus the calculated $\sin ^{2} \theta$ in the $\operatorname{stan}-$ dard 'run-and-match' approximation[7]. See eq. (3.13). Each point on this plot represents a specific choice of free parameters of the minimal supersymmetric $S U(5)$ grand-unified theory that survives experimental bounds on the spectrum. The parameter space spanned here is detailed in Table 1.

Fig. 9. Scatter plot of $\sin ^{2} \theta$ against the mass of the lightest chargino, $M_{C, \min }$, for the minimal supersymmetric $S U(5)$ grand-unified theory. Each point represents a choice of parameters; see Table 1.

Fig. 10. Scatter plot of $\sin ^{2} \theta$ against the mass of the lightest neutral Higgs, $M_{H, \min }$, for the minimal supersymmetric $S U(5)$ grand-unified theory. Each point represents a choice of parameters; see Table 1 .

Fig. 11. Scatter plot of $\sin ^{2} \theta$ against the mass of the lightest top-squark, $M_{T, \min }$, for the minimal supersymmetric $S U(5)$ grand-unified theory. Each point represents a choice of parameters; see Table 1.

Fig. 12. Scatter plot of $\sin ^{2} \theta$ against the mass of the lightest neutralino, $M_{N, \min }$, for the minimal supersymmetric $S U(5)$ grand-unified theory. Each point represents a choice of parameters; see Table 1. 


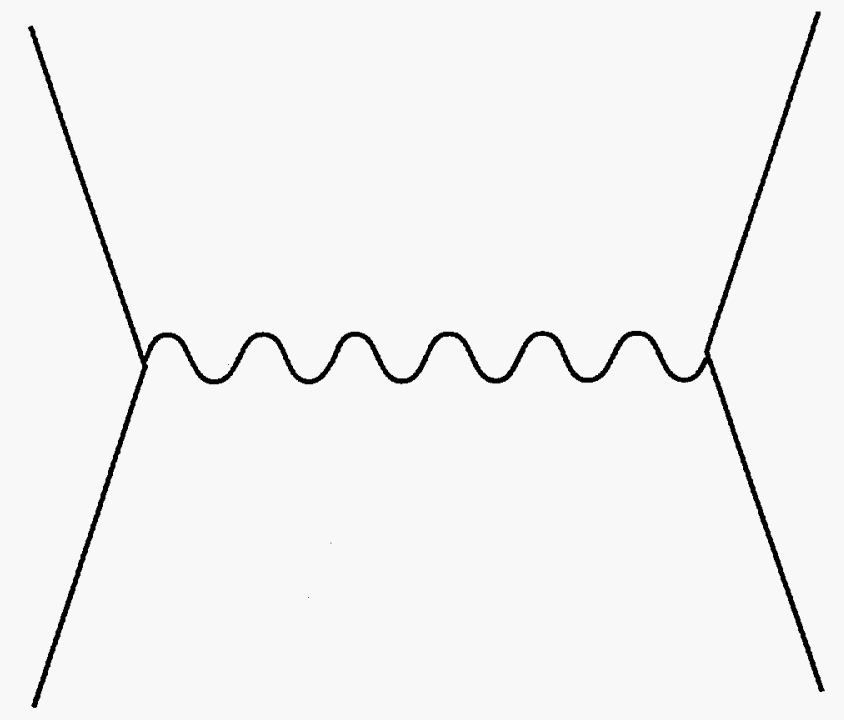

Fig 1

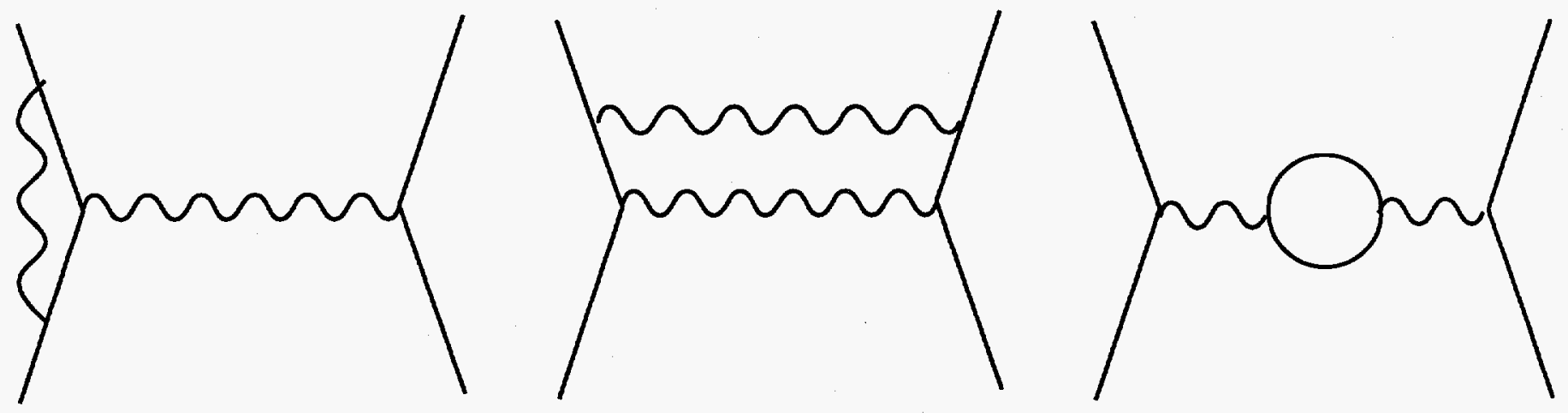

Fig 2 

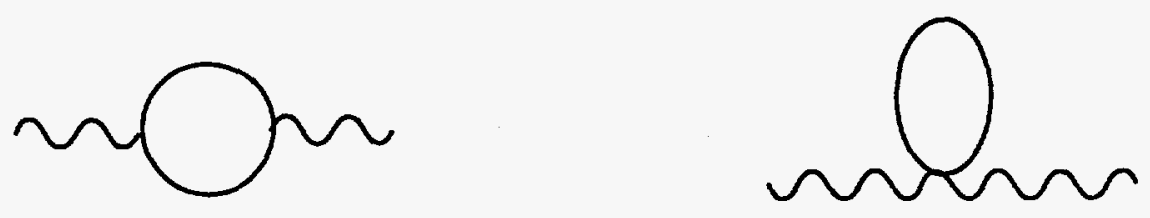

Fig 3

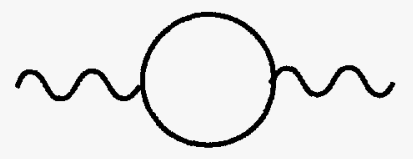

Fig 4 


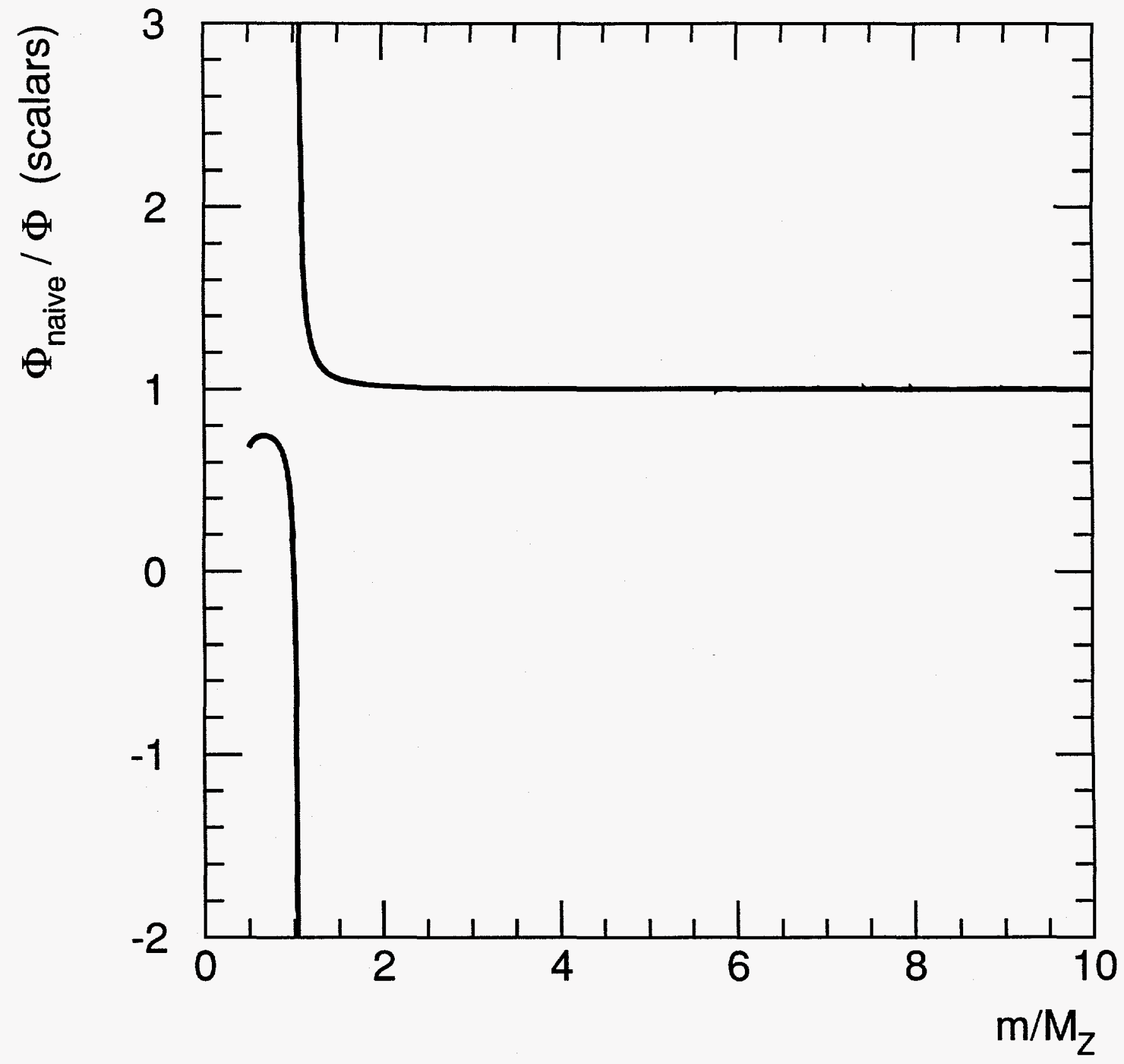

Fig. 5 


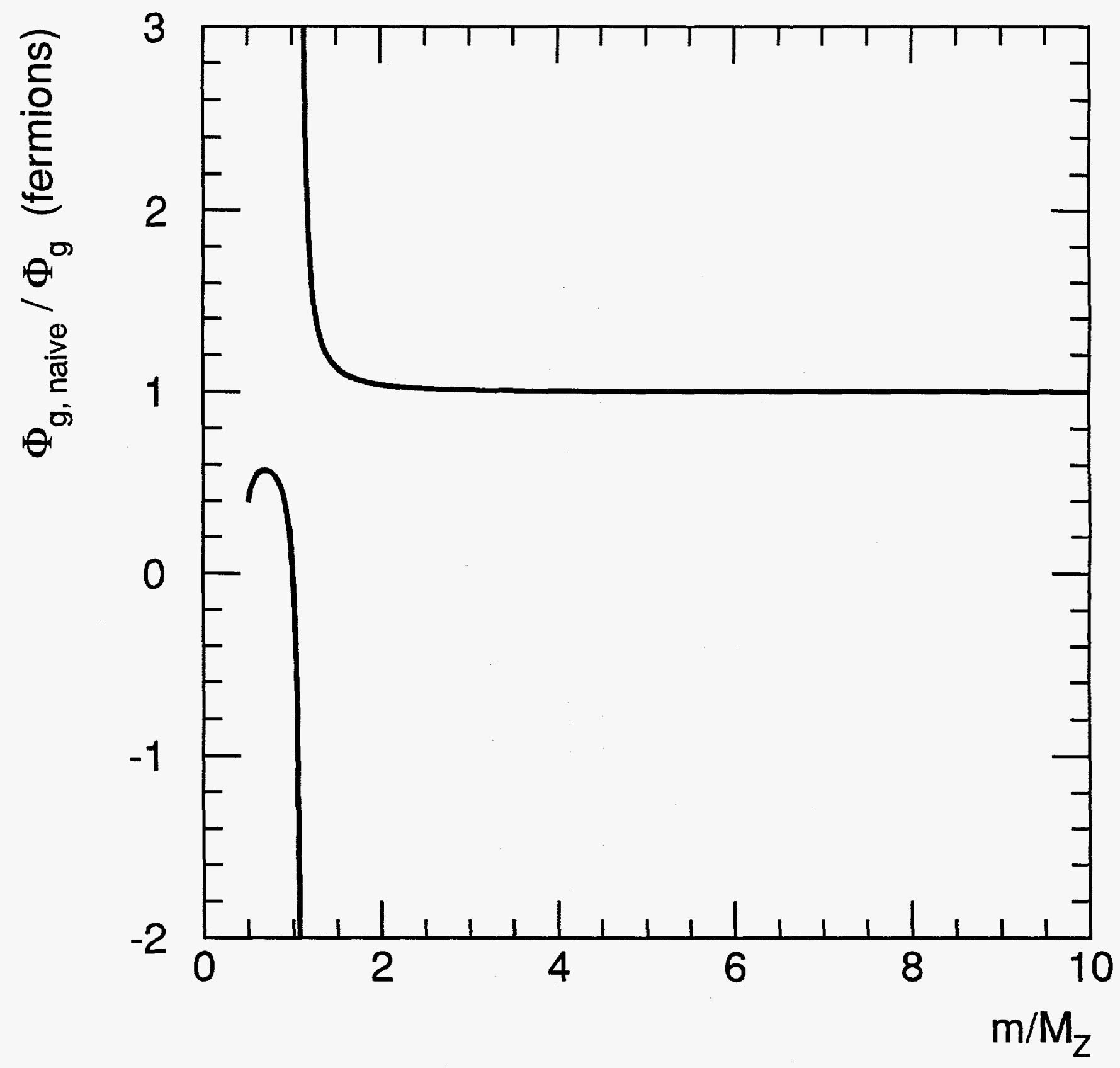

Fig. 6 


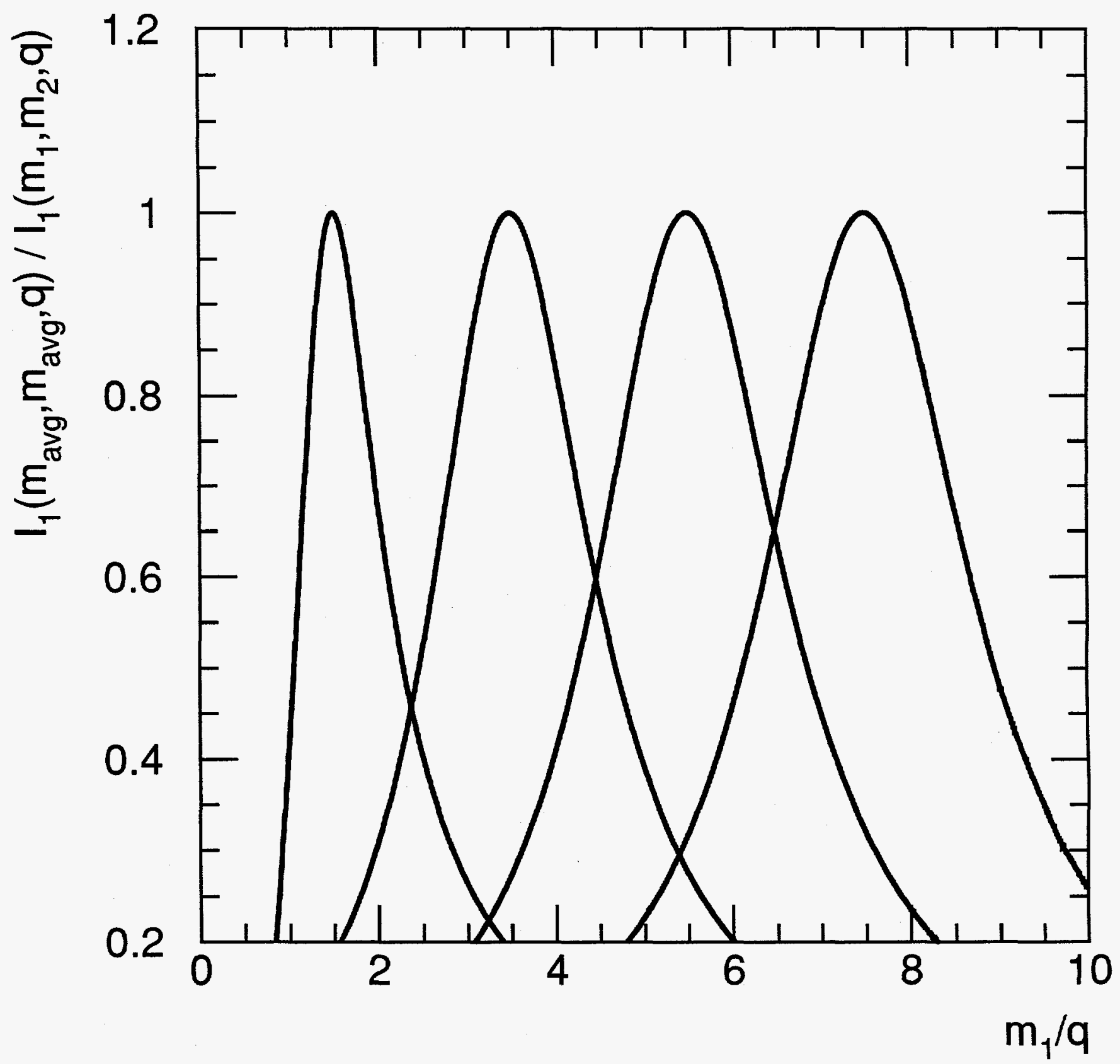

Fig. 7 


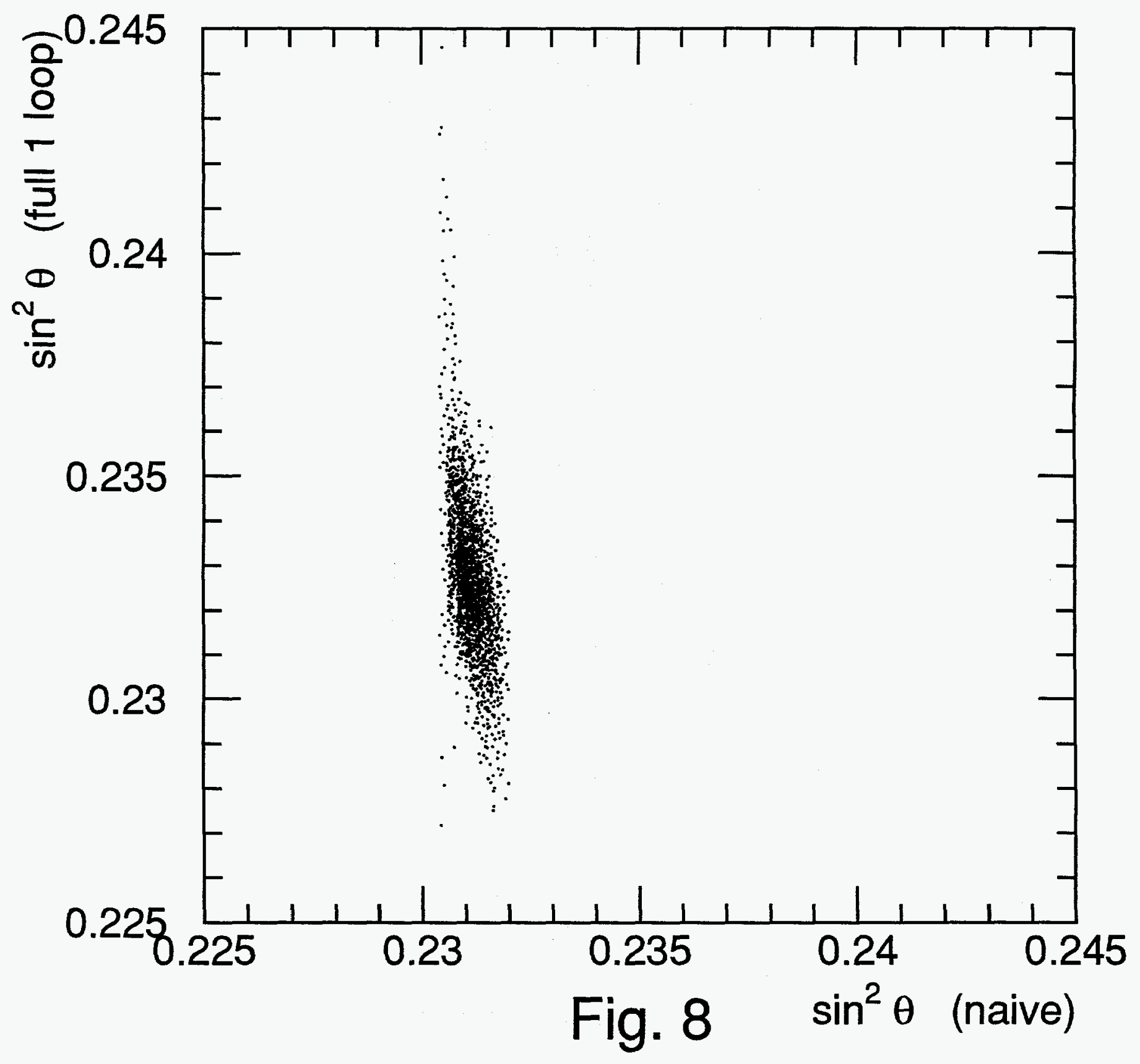




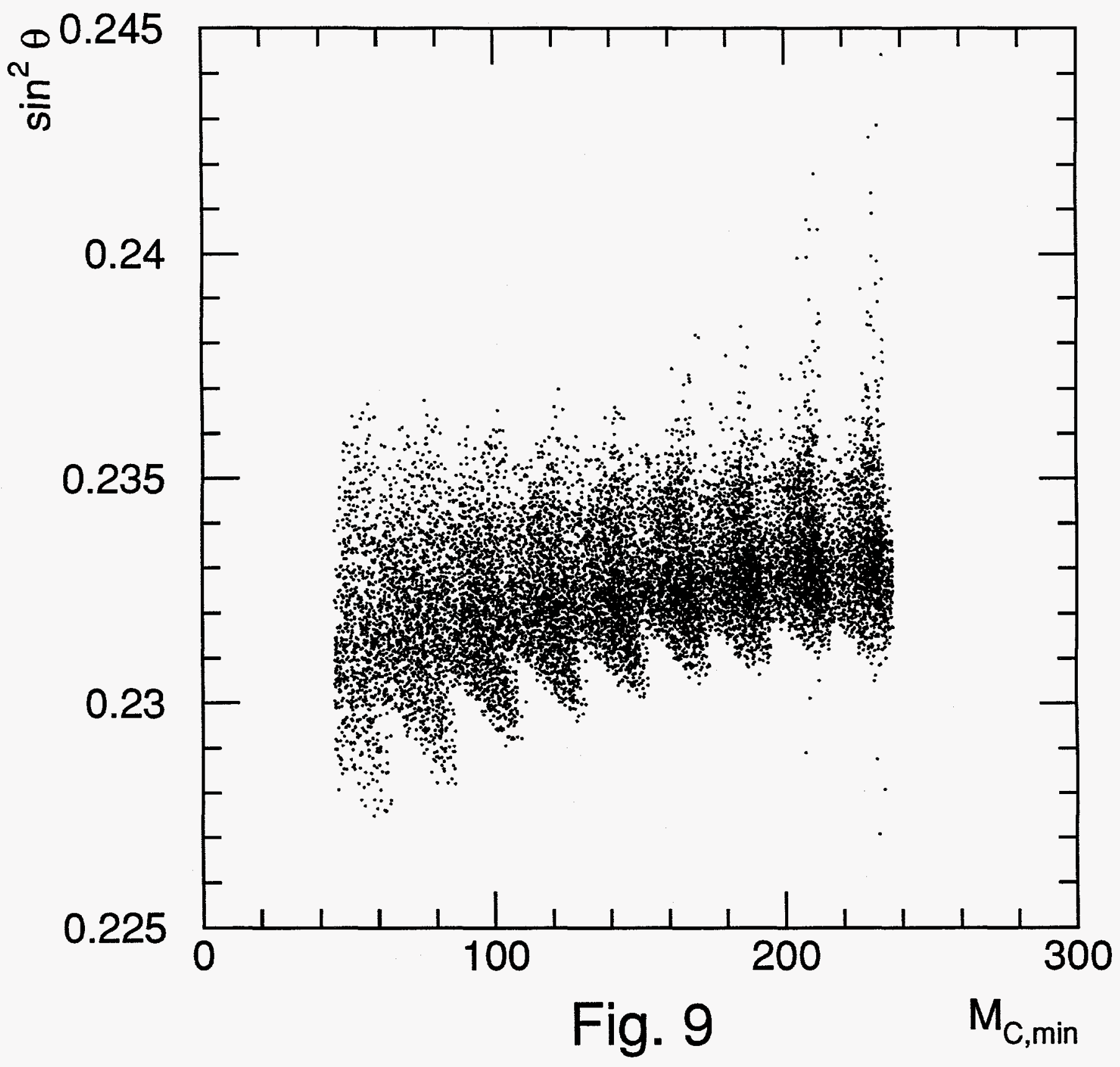




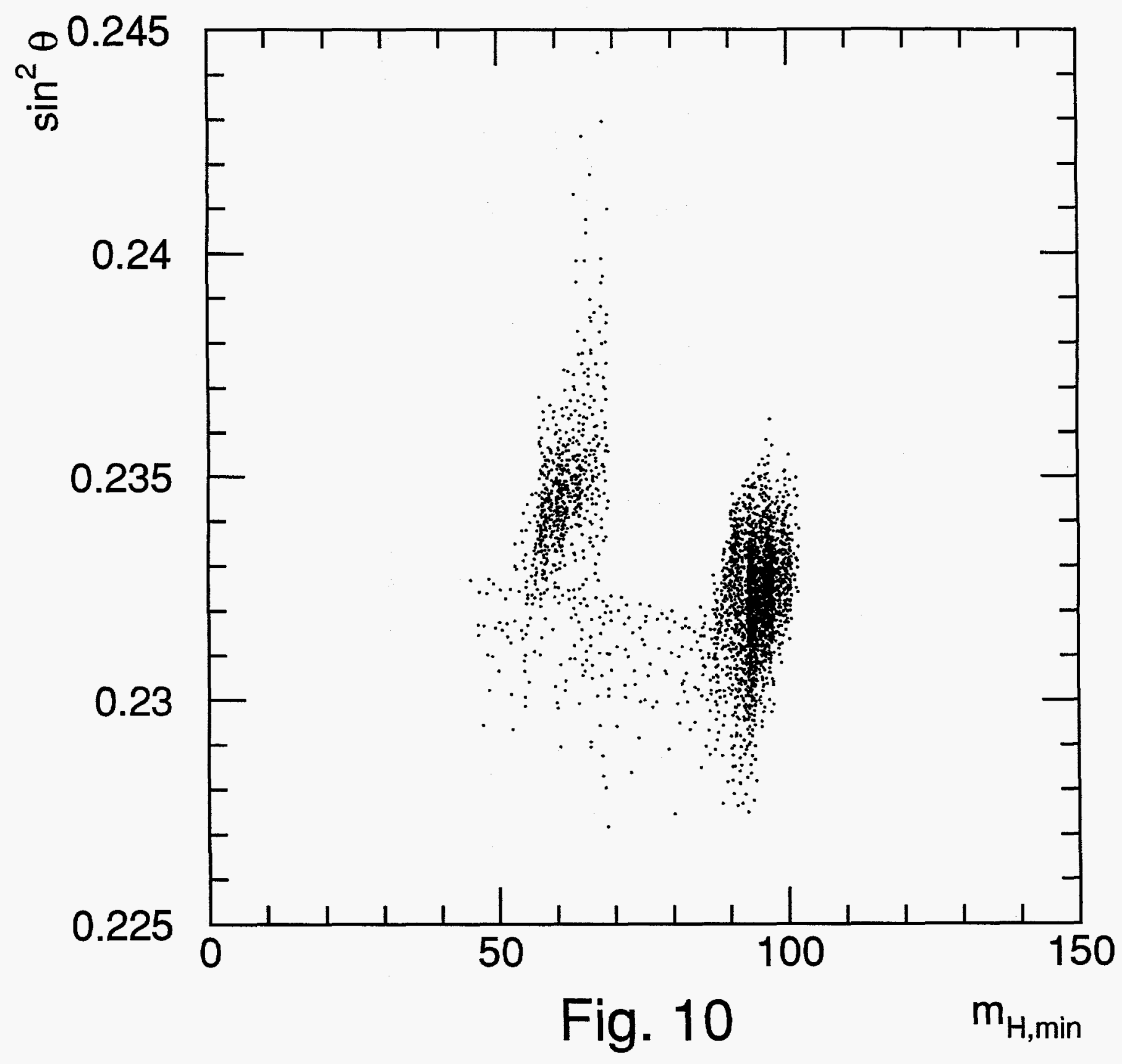




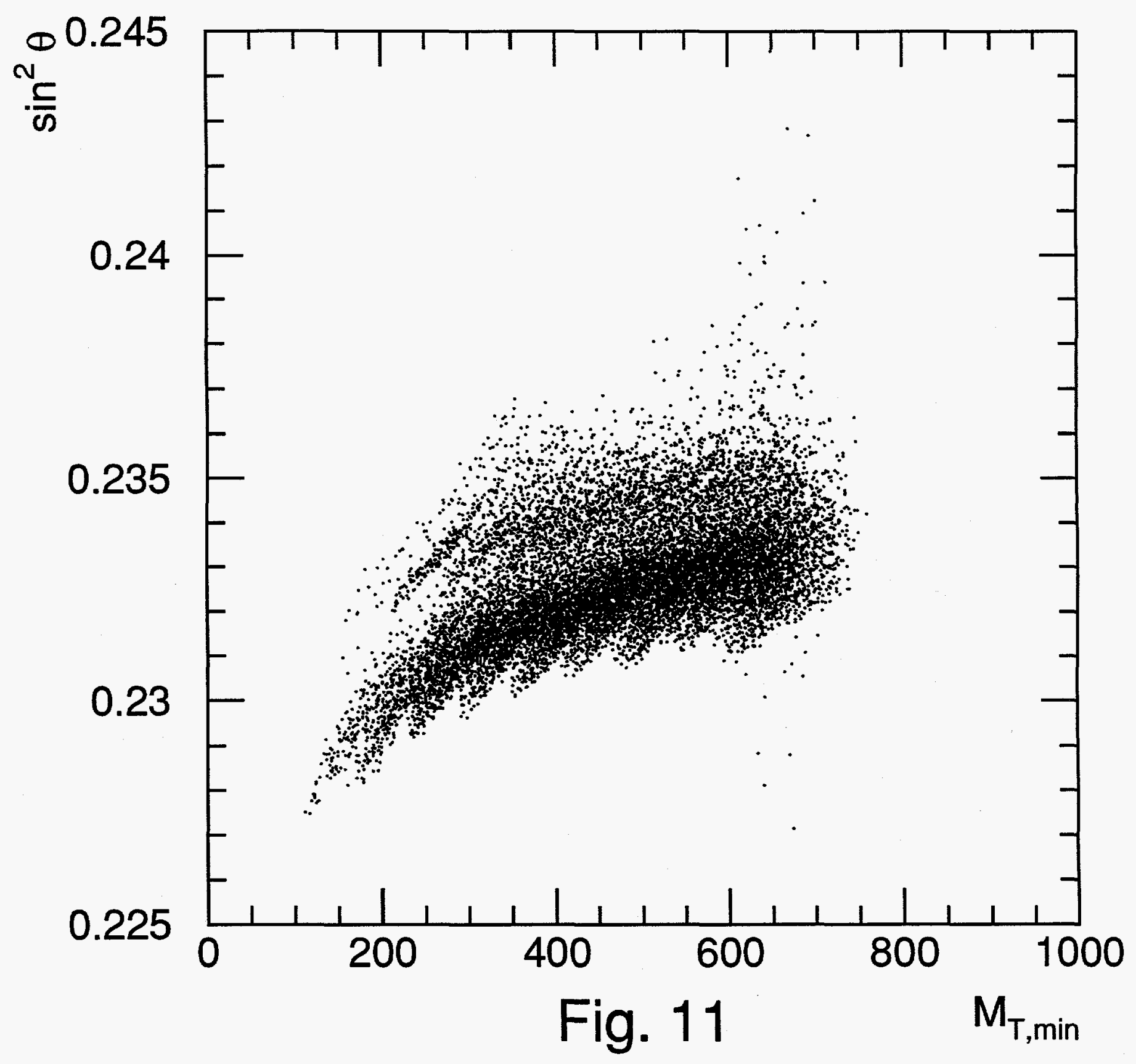




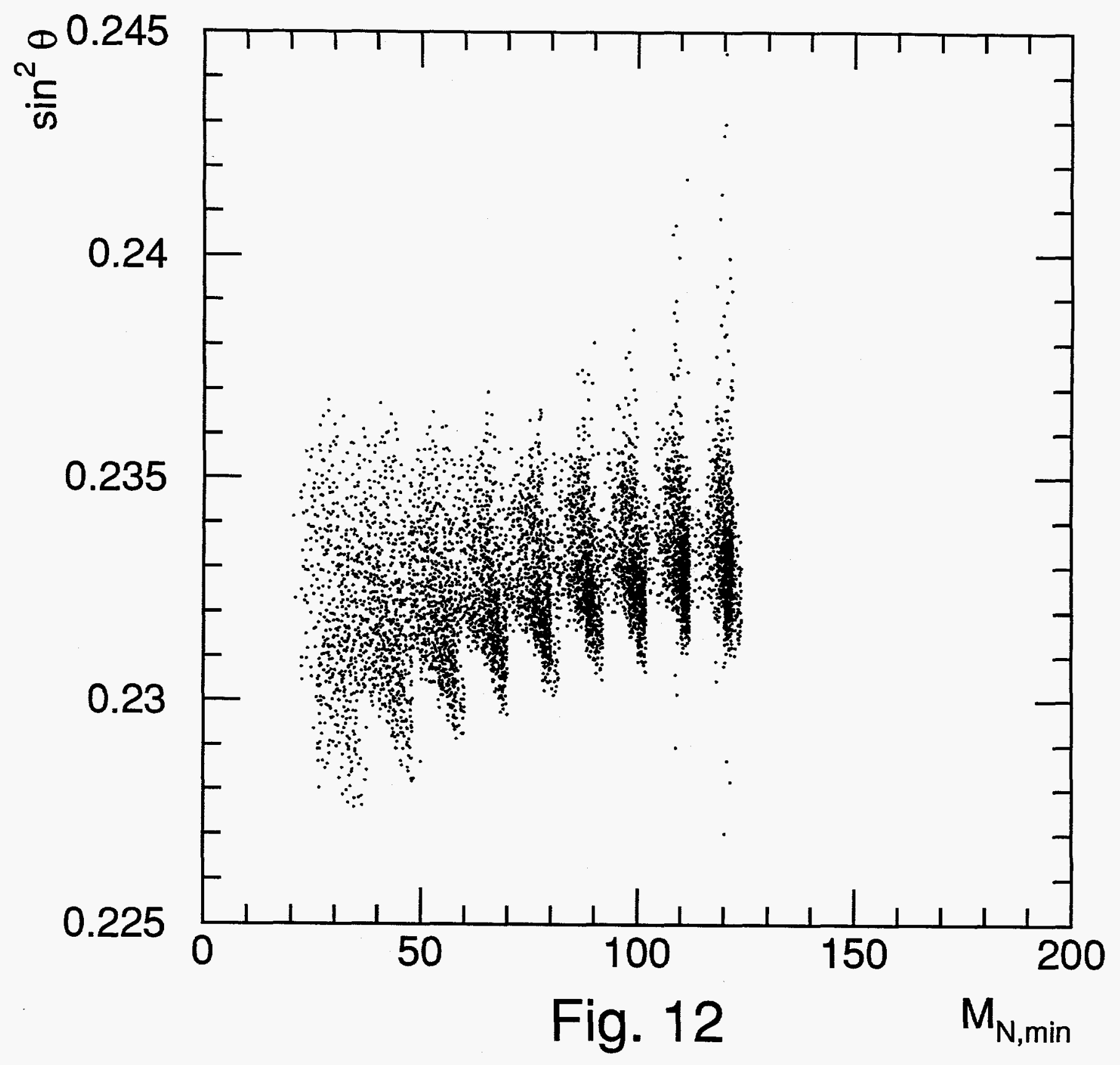

